\title{
Search for Dark Matter Particles Produced in Association with a Top Quark Pair at $\sqrt{s}=13$ TeV
}

\author{
A. M. Sirunyan et al. \\ (CMS Collaboration)
}

(Received 17 July 2018; published 10 January 2019)

\begin{abstract}
A search is performed for dark matter particles produced in association with a top quark pair in protonproton collisions at $\sqrt{s}=13 \mathrm{TeV}$. The data correspond to an integrated luminosity of $35.9 \mathrm{fb}^{-1}$ recorded by the CMS detector at the LHC. No significant excess over the standard model expectation is observed. The results are interpreted using simplified models of dark matter production via spin- 0 mediators that couple to dark matter particles and to standard model quarks, providing constraints on the coupling strength between the mediator and the quarks. These are the most stringent collider limits to date for scalar mediators, and the most stringent for pseudoscalar mediators at low masses.
\end{abstract}

DOI: 10.1103/PhysRevLett.122.011803

Astrophysical observations strongly motivate the existence of dark matter [1-4], which may originate from physics beyond the standard model. In a large class of models, dark matter consists of stable, weakly interacting massive particles $(\chi)$ [4], which may be pair produced at the CERN LHC via mediators that couple both to dark matter particles and to standard model quarks. The dark matter particles would escape detection, thereby creating a transverse momentum imbalance $\left(\vec{p}_{T}^{\text {miss }}\right)$ in the event. Searches at collider experiments can offer insights on the nature of the mediator and provide constraints on dark matter masses of $\mathcal{O}(10 \mathrm{GeV})$ and below, a region that is difficult to explore both in direct and indirect searches for dark matter. A favored class of models proposes a spin- 0 mediator with standard model Higgs-like Yukawa coupling to quarks, which therefore couples preferentially to the top quark [5-9]. Consequently, in this class of models dark matter production in association with a top quark pair $(t \bar{t})$ can offer better search sensitivity compared to other modes such as production in association with a jet [10-14]. At the LHC, the $t \bar{t}+\chi \bar{\chi}$ process is probed through the signature of $t \bar{t}$ accompanied by $\vec{p}_{T}^{\text {miss }}[15,16]$.

The top quark almost always decays to a $W$ boson and a $b$ quark. The $W$ boson can decay leptonically (to a charged lepton and a neutrino) or hadronically (to a quark pair). The signal regions (SRs) of the search cover three $t \bar{t}$ decay modes: the all-hadronic, lepton + jets $(\ell+$ jets where $\ell=e, \mu)$, and dileptonic $(e e, e \mu, \mu \mu)$ final states where

\footnotetext{
*Full author list given at the end of the Letter.

Published by the American Physical Society under the terms of the Creative Commons Attribution 4.0 International license. Further distribution of this work must maintain attribution to the author(s) and the published article's title, journal citation, and DOI. Funded by SCOAP ${ }^{3}$.
}

neither, either, or both of the $W$ bosons decay to leptons, respectively. This Letter presents a search for $t \bar{t}+\chi \bar{\chi}$ in $p p$ collisions at $\sqrt{s}=13 \mathrm{TeV}$ with data recorded by the CMS experiment in 2016, corresponding to an integrated luminosity of $35.9 \mathrm{fb}^{-1}$. The analysis strategy is similar to Ref. [17], but includes additional SRs for the dileptonic mode.

The central feature of the CMS detector is a superconducting solenoid providing a magnetic field of $3.8 \mathrm{~T}$. Within the solenoid volume are the silicon pixel and strip trackers, a lead tungstate crystal electromagnetic calorimeter, and a brass and scintillator hadron calorimeter. A steel and quartz-fiber Cherenkov forward hadron calorimeter extends the pseudorapidity $(\eta)$ coverage. The muon system consists of gas-ionization detectors embedded in the steel flux-return yoke outside the solenoid. A two-tiered trigger system [18] selects events at a rate of about $1 \mathrm{kHz}$ for storage. A detailed description of the CMS detector is provided in Ref. [19].

The event reconstruction is based on the CMS particleflow algorithm [20], which reconstructs and identifies individual particles using an optimized combination of the detector information. The $\vec{p}_{T}^{\text {miss }}$ vector is computed as the negative vector sum of the transverse momenta $\left(\vec{p}_{T}\right)$ of all the particles in an event. Jets are formed from particles using the anti- $k_{T}$ algorithm [21,22] with a distance parameter of 0.4. Corrections are applied to calibrate the jet momentum [23] and to remove energy from additional collisions in the same or adjacent bunch crossings (pileup) [24]. Jets in the analysis are required to have $p_{T}>30 \mathrm{GeV}$ and $|\eta|<2.4$, and to satisfy identification criteria [25] that minimize spurious detector effects. A combined secondary vertex $b$ tagging algorithm [26] is used to identify jets originating from $b$ quarks ( $b$-tagged jets). A multivariate discriminant, the "resolved top tagger" (RTT) [17], based 
on jet properties and kinematic information, is used to identify top quarks that decay into three jets. Electrons and muons are selected using "tight" and "loose" requirements where the former applies more stringent identification criteria than the latter [27]. The tight leptons are used in the selection of specific final states, while loose leptons are used to veto events with extra leptons. The primary $p p$ interaction vertex is taken to be the reconstructed vertex with the highest summed $p_{T}^{2}$ of its associated physics objects. Here, the physics objects are the jets, clustered with the tracks assigned to the vertex as inputs, plus the associated $\vec{p}_{T}^{\text {miss }}$.

The $t \bar{t}+\chi \bar{\chi}$ signal results in high- $p_{T}$ jets including $b$-tagged jets, leptons, and significant $\vec{p}_{T}^{\text {miss }}$. The background is dominated by $t \bar{t}$ and $V+$ jets $\left(V=W, Z / \gamma^{*}\right)$ production. The $t \bar{t}$ and single top quark backgrounds are simulated at next-to-leading order (NLO) accuracy in quantum chromodynamics (QCD) using POWHEG v2 and POWHEG v1 [28-31], respectively. Samples of $V+$ jets and QCD multijet events are simulated at leading order (LO) in QCD using MADGRAPH5_aMC@NLO v2.2.2 [32] (MADGRAPH), with up to four additional partons in the matrix element (ME) calculations. The $V+$ jets samples are corrected with boson $p_{T}$-dependent electroweak corrections [33-38] and QCD NLO/LO $K$ factors computed using MAdGraph. Samples of $t \bar{t}+V$ and diboson processes ( $W W, W Z$, and $Z Z)$ are generated at NLO using either MADGRAPH or POWHEG v2. The initial-state partons are modeled with the NNPDF 3.0 [39] parton distribution function (PDF) sets at LO or NLO in QCD to match the ME calculation. Generated events are interfaced with PYTHIA 8.205 [40] for parton showering using the CUETP8M1 tune [41], except for the $t \bar{t}$ simulation which uses the CUETP8M2 tune customized by CMS with an updated strong coupling $\alpha_{S}$ for initial-state radiation. The simulation of the CMS detector is performed with GEANT4 [42]. Corrections derived from data are applied to account for any mismodeling of selection efficiencies in simulation.

The signal is simulated using simplified models of dark matter production [43]. The dominant mechanism is $s$-channel production of the mediator via gluon fusion, with the mediator then decaying to a pair of dark matter particles. The dark matter particles are assumed to be Dirac fermions, and the mediators are spin- 0 particles with scalar $(\phi)$ or pseudoscalar $(a)$ interactions. The couplings between the mediator and standard model quarks are $g_{q q}=g_{q} y_{q}$, where $y_{q}=\sqrt{2} m_{q} / v$ are the standard model Yukawa couplings, $m_{q}$ is the quark mass, and $v=246 \mathrm{GeV}$ is the Higgs boson field vacuum expectation value. The $g_{q}$ parameter is assumed to be unity for all quarks. The direct coupling strength of the mediators to dark matter is denoted by $g_{\chi}$. The model does not take into account possible mixing between $\phi$ and the standard model Higgs boson [44]. The $t \bar{t}+\chi \bar{\chi}$ signal is generated at LO using MADGRAPH with up to one additional parton, and the mediator is forced to decay to a pair of dark matter fermions. The mediator width is computed according to partial-width formulas in Ref. [45] and assuming no additional interactions beyond those described here. The relative width of the scalar (pseudoscalar) mediator varies between $4 \%$ and $6 \%$ (4\% and $8 \%$ ) for masses in the range of $10-500 \mathrm{GeV}$. The signal is normalized to the cross section computed at NLO in QCD.

Data are collected by triggering on events containing large $p_{T}^{\text {miss }}$ (the magnitude of $\vec{p}_{T}^{\text {miss}}$ ) or high- $p_{T}$ leptons. The triggers for the all-hadronic final state are based on the amount of $p_{T}^{\text {miss }}$ and $H_{T}^{\text {miss }}$ measured with the trigger-level reconstruction. The $H_{T}^{\text {miss }}$ variable is defined as the magnitude of the vector sum of $\vec{p}_{T}$ over trigger-level jets with $p_{T}>20 \mathrm{GeV}$ and $|\eta|<5.0$ that pass identification requirements. During the period of data collection, the $p_{T}^{\text {miss }}$ and $H_{T}^{\text {miss }}$ trigger thresholds were increased as the instantaneous luminosity increased, in steps from 90 to $120 \mathrm{GeV}$. Events in the $\ell+$ jets final state are obtained using single-lepton triggers that require an electron (muon) with $p_{T}>27$ (24) GeV. Events in the dilepton final state are obtained using single-lepton and dilepton $(e e, e \mu, \mu \mu)$ triggers. The trigger thresholds on the higher- and lower- $p_{T}$ electrons (muons) are 23 (17) $\mathrm{GeV}$ and 12 (8) GeV, respectively, and apply to all pairings of lepton object flavors.

Using additional selection requirements, two allhadronic, one $\ell+$ jets, and four dilepton SRs are defined. Several control regions (CRs) enriched in standard model processes are used to improve the simulation-based background estimates for the all-hadronic and $\ell+$ jets SRs. There are no event overlaps among the regions. Together, the SRs and CRs associated with the individual $t \bar{t}+\chi \bar{\chi}$ final states are referred to as "channels." All three $t \bar{t}+\chi \bar{\chi}$ channels are used in a simultaneous maximum-likelihood fit of $p_{T}^{\text {miss }}$ distributions to extract a potential dark matter signal. In the fit, the CRs constrain the contributions of $t \bar{t}$, $W+$ jets, and $Z+$ jets processes within each channel via freely floating normalization parameters for each $p_{T}^{\text {miss }}$ bin.

The all-hadronic SRs require $p_{T}^{\text {miss }}>200 \mathrm{GeV}$, and four or more jets, of which at least one must be $b$ tagged. Any event with a loose lepton of $p_{T}>10 \mathrm{GeV}$ is vetoed. The dominant background consists of $t \bar{t}$ decays to $\ell+$ jets, referred to as $t \bar{t}(1 \ell)$, where the lepton is not identified as loose and therefore not vetoed, and the neutrino is the source of $\vec{p}_{T}^{\text {miss }}$. The RTT is employed to define a category of events with two tagged hadronic top quark decays (2RTT), which suppresses the $t \bar{t}(1 \ell)$ background, and a category with less than two top quark tags $(0,1 \mathrm{RTT})$ and at least two $b$-tagged jets. The RTT variable is essentially independent of $p_{T}^{\text {miss }}$; therefore, any bias on the $p_{T}^{\text {miss }}$ shape from requiring top tags is negligible. Spurious $p_{T}^{\text {miss }}$ can arise in multijet events as a result of jet energy mismeasurement. In such cases, the reconstructed $\vec{p}_{T}^{\text {miss }}$ tends to align with a jet. The multijet background is suppressed by 
requiring the smallest azimuthal angle between the $\vec{p}_{T}^{\text {miss }}$ and each jet in the event, $\Delta_{j} \equiv \min \Delta \phi\left(\vec{p}_{T}^{\mathrm{j}}, \vec{p}_{T}^{\text {miss }}\right)$, to be greater than 0.4 (1.0) radians in the 2RTT $(0,1 \mathrm{RTT})$ category. The $\Delta_{j}$ requirement also reduces the $t \bar{t}(1 \ell)$ background, for which $\vec{p}_{T}^{\text {miss }}$ can align with a bottom jet.

The CRs targeting the $t \bar{t}(1 \ell)$ background, one for each category, are defined by selecting events with exactly one tight lepton with $p_{T}>30 \mathrm{GeV}$, and by requiring the transverse mass, $m_{T}$, given in terms of $\vec{p}_{T}^{\text {miss }}$ and the lepton momentum $\left(\vec{p}_{T}^{\ell}\right)$ by the following expression:

$$
m_{T}=\sqrt{2 p_{T}^{\ell} p_{T}^{\text {miss }}\left[1-\cos \Delta \phi\left(\vec{p}_{T}^{\ell}, \vec{p}_{T}^{\text {miss }}\right)\right]},
$$

to be less than $160 \mathrm{GeV}$, in order to avoid overlaps with the SR of the $\ell+$ jets channel. For ideal measurements, the $m_{T}$ quantity is bounded above by the $W$ boson mass for $t \bar{t}(1 \ell)$ and for $W(\ell \nu)+$ jets where the $W$ boson is produced on shell.

There are also significant background contributions from $Z(\nu \bar{\nu})+$ jets, and from $W(\ell \nu)+$ jets where the lepton is not identified. The CRs enriched in both $W+$ jets and $Z+$ jets are formed by modifying the SR selections to require no $b$-tagged jets. Additionally, dedicated $W+$ jets CRs are defined by requiring a tight lepton with $p_{T}>$ $30 \mathrm{GeV}$ and $m_{T}<160 \mathrm{GeV}$. A CR enriched in $Z+$ jets is defined by selecting two tight, oppositely charged, and same-flavor leptons, with the dilepton invariant mass between 60 and $120 \mathrm{GeV}$. This CR is not subdivided into categories based on the number of top quark tags because the event yield with two tags is negligible. The $p_{T}^{\text {miss }}$ calculation does not consider the two leptons in order to emulate the $Z(\nu \bar{\nu})+$ jets process.

Events in the $\ell+$ jets SR are selected by requiring $p_{T}^{\text {miss }}>160 \mathrm{GeV}$, exactly one tight lepton with $p_{T}>30 \mathrm{GeV}$, and three or more jets, of which at least one is $b$ tagged. Events must not contain additional loose leptons with $p_{T}>10 \mathrm{GeV}$. A selection of $m_{T}>160 \mathrm{GeV}$ is imposed to reduce the $t \bar{t}(1 \ell)$ and $W+$ jets backgrounds. Following these selections, the remaining background events primarily consist of dileptonic $t \bar{t}$ decays, referred to as $t \bar{t}(2 \ell)$ events, where one of the leptons is not identified. This background is suppressed by requiring that the $m_{T 2}^{W}$ quantity [46] be larger than $200 \mathrm{GeV}$, and for the two highest $p_{T}$ jets in the event that $\Delta_{j_{1,2}} \equiv$ $\min \Delta \phi\left(\vec{p}_{T}^{\mathrm{j}_{1,2}}, \vec{p}_{T}^{\mathrm{miss}}\right)>1.2$. The $m_{T 2}^{W}$ variable corresponds to the minimum mass of a particle consistent with being pair-produced and decaying to a bottom quark and a $W$ boson, where both $W$ bosons decay leptonically but one of the two leptons is not detected. The key characteristic of $m_{T 2}^{W}$ is that for ideal measurements the distribution for $t \bar{t}(2 \ell)$ events is bounded above by the top quark mass.

The CR targeting the $t \bar{t}(2 \ell)$ background is defined by requiring an additional tight lepton of $p_{T}>30 \mathrm{GeV}$ with respect to the SR selection and removing the selections on $m_{T}, m_{T 2}^{W}$, and $\Delta_{j_{1,2}}$. To reduce the signal contamination and avoid overlap with the dileptonic SRs, the $m_{T 2}^{\ell \ell}$ variable [47-49] is required to be less than $110 \mathrm{GeV}$. The $m_{T 2}^{\ell \ell}$ variable is essentially the minimum $m_{T}$ of a pairproduced particle that decays to a lepton and a neutrino. The $m_{T 2}^{\ell \ell}$ distribution for $t \bar{t}(2 \ell)$ events is bounded above by the $W$ boson mass for ideal measurements. A $W+$ jets CR for the $\ell+$ jets channel is defined by requiring no $b$-tagged jets and removing the selections on $m_{T 2}^{W}$ and $\Delta_{\mathrm{j}_{1,2}}$.

Events in the dilepton SRs are selected by requiring exactly two oppositely charged tight leptons with higher (lower) $p_{T}>25$ (15) GeV, two or more jets with at least one $b$-tagged jet, and $p_{T}^{\text {miss }}>50 \mathrm{GeV}$. The dilepton mass is required to be greater than $20 \mathrm{GeV}$, and for the dielectron and dimuon events, to be at least $15 \mathrm{GeV}$ away from the $Z$ boson pole mass $\left(m_{Z}\right)$ [50]. Separate categories are considered for events with same- and different-flavor lepton pairs, and for events with $m_{T 2}^{\ell \ell}$ greater or less than $110 \mathrm{GeV}$. Additionally, events with $m_{T 2}^{\ell \ell}<110 \mathrm{GeV}$ must not pass the selection for the $t \bar{t}(2 \ell) \mathrm{CR}$ of the $\ell+$ jets search channel. The SRs with large $m_{T 2}^{\ell \ell}$ have significantly higher signal purity.

The estimates for the backgrounds from Drell-Yan production and from jets misidentified as leptons are performed using dedicated sideband regions not included in the fit. A $p_{T}^{\text {miss }}$-dependent correction to the Drell-Yan simulation in the same-flavor SRs is obtained by comparing data and simulation yields within $15 \mathrm{GeV}$ of $m_{Z}$. The misidentified leptons background is estimated using data events with pairs consisting of one tight lepton plus a "nontight" lepton-like object passing a less stringent selection. The number of such combinations is scaled by misidentification rates, which are measured in a jetenriched sample.

The dark matter signal, which would be observed as an excess of events compared to the predicted background at high $p_{T}^{\text {miss }}$, is extracted via a simultaneous maximumlikelihood fit to the binned $p_{T}^{\text {miss }}$ distributions of the signal and backgrounds, based on simulation with the exception of the misidentified leptons background, in the SRs and associated CRs. The fit is performed using the RoOSTATS statistical package [51]. The template shapes and normalization are allowed to vary in the fit, constrained by the priors of the systematic uncertainties, parametrized as nuisance parameters.

The common sources of uncertainty are correlated across SRs and CRs and across channels. The sources of normalization uncertainty include the integrated luminosity (2.5\%) [52], $b$ tagging efficiency (1\%-5\%) [26], lepton efficiency $(1.5 \%-2.2 \%)$ [27], and pileup simulation $(0.8 \%-1.9 \%)$, where the range of values indicates variations across different physics processes. The common sources of shape uncertainty include $p_{T}^{\text {miss }}$ trigger efficiency, jet energy scale 
and resolution [25], PDF [39], uncertainty on the $K$ factors for $V+$ jets, uncertainty from missing higher order QCD corrections for each simulated physics process, and the uncertainty in the modeling of top quark $p_{T}$ in $t \bar{t}$ simulation [53]. The jet energy scale uncertainties have the largest impact on the $\ell+$ jets and dilepton channels, while in the all-hadronic channel the top quark $p_{T}$ modeling and $p_{T}^{\text {miss }}$ trigger uncertainties are more important.

Within the all-hadronic and $\ell+$ jets search channels, additional nuisance parameters scale the yields of the $t \bar{t}$, $W+$ jets, and $Z+$ jets backgrounds independently in each $p_{T}^{\text {miss }}$ bin across the SRs and CRs of a given channel. For example, in each bin of $p_{T}^{\text {miss }}$ a single parameter is associated with the contribution of the $W+$ jets process in the all-hadronic SRs and CRs, while another set of parameters distinct from those of the all-hadronic channel, is associated with the $W+$ jets background in the $\ell+$ jets SRs and CRs. These nuisance parameters allow the data in the CRs to constrain the estimates of the dominant background processes in the corresponding SRs. Signal yields in all the SRs and CRs are scaled simultaneously by the signal strength parameter $(\mu)$, defined as the ratio of the measured signal cross section to the theoretical cross section, $\mu=\sigma / \sigma_{\text {th }}$.

The fit is performed across all search channels and no significant excess is observed. Figure 1 shows the $p_{T}^{\text {miss }}$ distributions for three of the seven SRs, obtained after the background-only fit assuming the absence of any signal. Upper limits are set on the $t \bar{t}+\chi \bar{\chi}$ production cross section using a modified frequentist approach $\left(\mathrm{CL}_{\mathrm{s}}\right)$ with a test statistic based on the profile likelihood in the asymptotic approximation [54-56]. For each signal hypothesis, 95\% confidence level (C.L.) upper limits on $\mu$ are determined. The all-hadronic channel provides the best sensitivity. The dileptonic channel is competitive with the all-hadronic channel for scalar mediator masses less than about $50 \mathrm{GeV}$, where the signal has a soft $p_{T}^{\text {miss }}$ spectrum, but is typically the least sensitive channel in other regions of the parameter space.

The limits are shown as a function of $m_{a / \phi}$ and $m_{\chi}$ in Fig. 2. The contours enclose the region where the upper limit on $\mu$ is less than 1 . Because of the narrow width of the mediator, the signal cross section drops rapidly across the $m_{a / \phi}=2 m_{\chi}$ line, marking the boundary between the onshell to the off-shell region. Therefore, the exclusion contour runs close to the $m_{a / \phi}=2 m_{\chi}$ line but does not cross it. The observed (expected) upper limits on $\mu$ exclude scalar and pseudoscalar masses of $160(240)$ and 220 (320) $\mathrm{GeV}$, respectively, at $95 \%$ C.L. The observed exclusion is weaker than the expected because of tension in the fit between CRs and SRs of the all-hadronic channel, although the difference is not significant as the observed result lies only just outside the $68 \%$ probability interval.
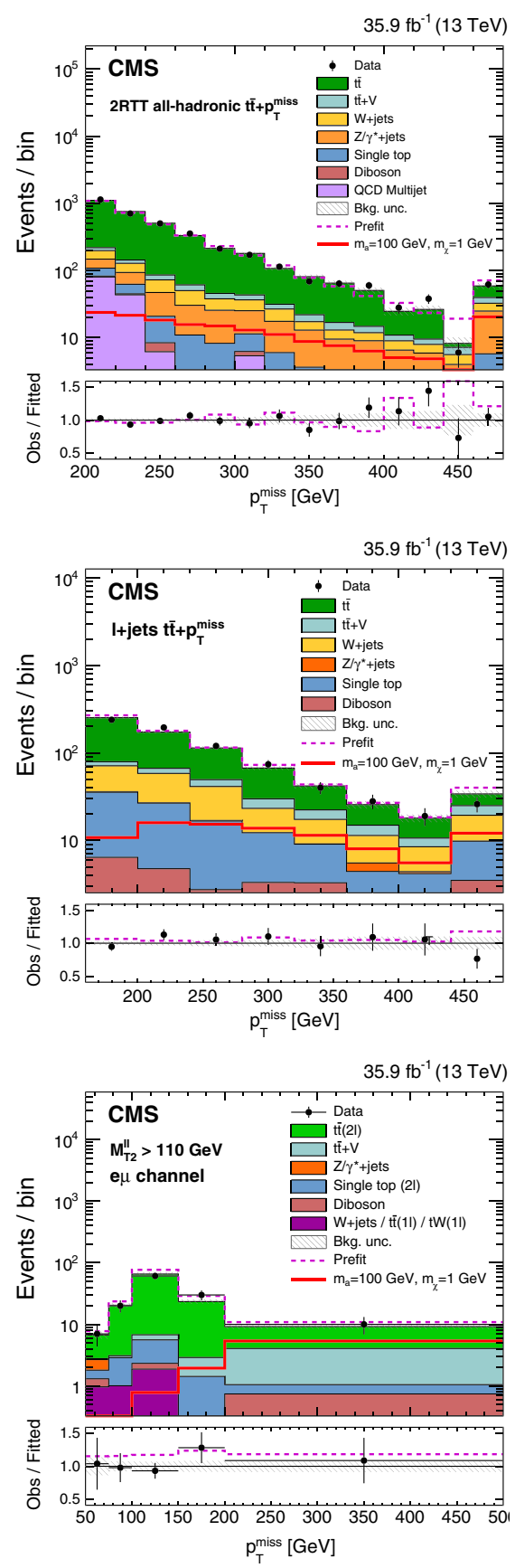

FIG. 1. Selected $p_{T}^{\text {miss }}$ distributions in SRs: 2RTT SR for the all-hadronic (top), the $\ell+$ jets (middle), and the differentflavor, $m_{T 2}^{\ell \ell}>110 \mathrm{GeV} \mathrm{SR}$ in the dileptonic channel (bottom). The solid red line shows the expectation for a signal with $m_{a}=$ $100 \mathrm{GeV}$ and $m_{\chi}=1 \mathrm{GeV}$. The last bin contains the overflow events. The lower panel shows the ratio of the observed to the fitted distribution (points), and the ratio of the background expectation before the fit to the fitted distribution (dashed magenta line). The vertical bars indicate the statistical uncertainty on the data. The horizontal bars on the rightmost plot indicate the bin width. The uncertainty bands in both panels include the statistical and systematic uncertainties on the total background. 

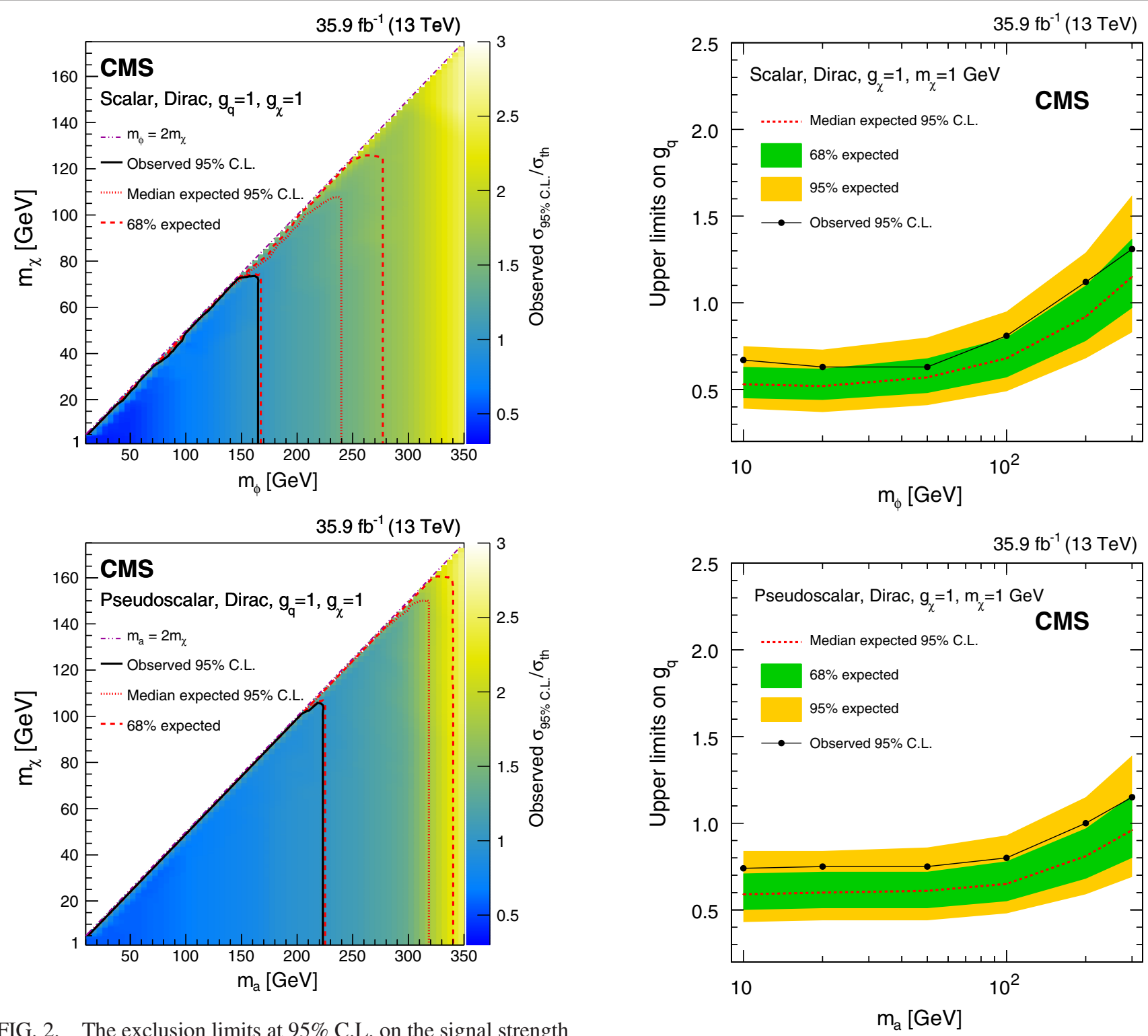

FIG. 2. The exclusion limits at $95 \%$ C.L. on the signal strength $\mu=\sigma / \sigma_{\text {th }}$ computed as a function of the mediator and dark matter mass, assuming a scalar (top) and pseudoscalar (bottom) mediator. The mediator couplings are assumed to be $g_{q}=g_{\chi}=1$. The dashed magenta lines represent the $68 \%$ probability interval around the expected limit. The observed limit contour is almost coincident with the boundary of the $68 \%$ probability interval.

This arises because the a priori estimation of the background exceeds the number of events observed in the CRs, while the estimate is in better agreement with data in the SRs. Consequently, the signal + background fit, in contrast to the background-only fit, reduces this tension between CRs and SRs by accommodating for some signal, which contributes primarily to the SRs.

The limits on $\mu$ are also expressed in terms of the mediator coupling strength to quarks in Fig. 3. These results are obtained by fixing $m_{\chi}=1 \mathrm{GeV}$ and $g_{\chi}=1$, and then finding the value of $g_{q}$ that corresponds to the upper limit on the cross section. This procedure is valid because

FIG. 3. The $95 \%$ observed and median expected C.L. upper limits on the coupling strength of the mediator to the standard model quarks under the assumption that $g_{\chi}=1$. A dark matter particle with a mass of $1 \mathrm{GeV}$ is assumed. The green and yellow bands indicate respectively the $68 \%$ and $95 \%$ probability intervals around the expected limit. The interpretations for a scalar (top) and a pseudoscalar (bottom) mediator are shown.

the kinematic properties of the signal do not vary appreciably with $g_{q}$. The width-to-mass ratio is around $4 \%$ for the $g_{q}$ and $m_{a / \phi}$ values considered.

In summary, a comprehensive search for dark matter particles produced in association with a top quark pair yields no significant excess over the predicted background. The results presented in this Letter provide 30\%-60\% better cross section limits compared to earlier searches targeting the same signature [57-59]. The analysis offers stronger constraints than direct and indirect experiments 
for dark matter masses of $\mathcal{O}(10 \mathrm{GeV})$ and below. Over much of the parameter space, the $t \bar{t}+\chi \bar{\chi}$ signature has better sensitivity for spin- 0 mediators than dark matter production in association with a jet [14] - previously considered to be the most sensitive signature. For the pseudoscalar model, the $t \bar{t}+\chi \bar{\chi}$ signature provides the most stringent cross section constraints for mediator masses of around $200 \mathrm{GeV}$ and below. The observed (expected) limits exclude a pseudoscalar mediator with mass below 220 (320) GeV under the $g_{q}=g_{\chi}=1$ benchmark scenario. The $t \bar{t}+\chi \bar{\chi}$ signature provides the best sensitivity for the scalar mediator model and is currently the only collider signature that is sufficiently sensitive to exclude regions of parameter space with these values of the couplings. The observed exclusion of a mediator with mass below $160 \mathrm{GeV}$ (240 GeV expected) provides the most stringent constraint to date on this model.

We congratulate our colleagues in the CERN accelerator departments for the excellent performance of the LHC and thank the technical and administrative staffs at CERN and at other CMS institutes for their contributions to the success of the CMS effort. In addition, we gratefully acknowledge the computing centers and personnel of the Worldwide LHC Computing Grid for delivering so effectively the computing infrastructure essential to our analyses. Finally, we acknowledge the enduring support for the construction and operation of the LHC and the CMS detector provided by the following funding agencies: BMWFW and FWF (Austria); FNRS and FWO (Belgium); CNPq, CAPES, FAPERJ, and FAPESP (Brazil); MES (Bulgaria); CERN; CAS, MoST, and NSFC (China); COLCIENCIAS (Colombia); MSES and CSF (Croatia); RPF (Cyprus); SENESCYT (Ecuador); MoER, ERC IUT, and ERDF (Estonia); Academy of Finland, MEC, and HIP (Finland); CEA and CNRS/IN2P3 (France); BMBF, DFG, and HGF (Germany); GSRT (Greece); OTKA and NIH (Hungary); DAE and DST (India); IPM (Iran); SFI (Ireland); INFN (Italy); MSIP and NRF (Republic of Korea); LAS (Lithuania); MOE and UM (Malaysia); BUAP, CINVESTAV, CONACYT, LNS, SEP, and UASLP-FAI (Mexico); MBIE (New Zealand); PAEC (Pakistan); MSHE and NSC (Poland); FCT (Portugal); JINR (Dubna); MON, RosAtom, RAS, RFBR and RAEP (Russia); MESTD (Serbia); SEIDI and CPAN (Spain); Swiss Funding Agencies (Switzerland); MST (Taipei); ThEPCenter, IPST, STAR, and NSTDA (Thailand); TUBITAK and TAEK (Turkey); NASU and SFFR (Ukraine); STFC (United Kingdom); DOE and NSF (USA).

[1] G. Bertone, D. Hooper, and J. Silk, Particle dark matter: Evidence, candidates and constraints, Phys. Rep. 405, 279 (2005).
[2] J. L. Feng, Dark matter candidates from particle physics and methods of detection, Annu. Rev. Astron. Astrophys. 48, 495 (2010).

[3] T. A. Porter, R. P. Johnson, and P.W. Graham, Dark matter searches with astroparticle data, Annu. Rev. Astron. Astrophys. 49, 155 (2011).

[4] G. Bertone, N. Bozorgnia, J. S. Kim, S. Liem, C. McCabe, S. Otten, and R. Ruiz de Austri, Identifying WIMP dark matter from particle and astroparticle data, J. Cosmol. Astropart. Phys. 03 (2018) 026.

[5] U. Haisch, F. Kahlhoefer, and J. Unwin, The impact of heavy-quark loops on LHC dark matter searches, J. High Energy Phys. 07 (2013) 125.

[6] T. Lin, E. W. Kolb, and L.-T. Wang, Probing dark matter couplings to top and bottom quarks at the LHC, Phys. Rev. D 88, 063510 (2013).

[7] M. R. Buckley, D. Feld, and D. Gonçalves, Scalar simplified models for dark matter, Phys. Rev. D 91, 015017 (2015).

[8] U. Haisch and E. Re, Simplified dark matter top-quark interactions at the LHC, J. High Energy Phys. 06 (2015) 078.

[9] C. Arina, M. Backović, E. Conte, B. Fuks, J. Guo, J. Heisig, B. Hespel, M. Krämer, F. Maltoni, A. Martini, K. Mawatari, M. Pellen, and E. Vryonidou, A comprehensive approach to dark matter studies: Exploration of simplified top-philic models, J. High Energy Phys. 11 (2016) 111.

[10] ATLAS Collaboration, Search for new phenomena in final states with an energetic jet and large missing transverse momentum in pp collisions at $\sqrt{s}=8 \mathrm{TeV}$ with the ATLAS detector, Eur. Phys. J. C 75, 299 (2015); Erratum, Eur. Phys. J. C 75, 408 (2015).

[11] CMS Collaboration, Search for dark matter in proton-proton collisions at $8 \mathrm{TeV}$ with missing transverse momentum and vector boson tagged jets, J. High Energy Phys. 12 (2016) 083.

[12] ATLAS Collaboration, Search for new phenomena in final states with an energetic jet and large missing transverse momentum in $\mathrm{pp}$ collisions at $\sqrt{s}=13 \mathrm{TeV}$ using the ATLAS detector, Phys. Rev. D 94, 032005 (2016).

[13] CMS Collaboration, Search for dark matter produced with an energetic jet or a hadronically decaying $\mathrm{W}$ or $\mathrm{Z}$ boson at $\sqrt{s}=13 \mathrm{TeV}$, J. High Energy Phys. 07 (2017) 14.

[14] CMS Collaboration, Search for new physics in final states with an energetic jet or a hadronically decaying $W$ or $Z$ boson and transverse momentum imbalance at $\sqrt{s}=13 \mathrm{TeV}$, Phys. Rev. D 97, 092005 (2018).

[15] CMS Collaboration, Search for the production of dark matter in association with top-quark pairs in the singlelepton final state in proton-proton collisions at $\sqrt{s}=8 \mathrm{TeV}$, J. High Energy Phys. 06 (2015) 121.

[16] ATLAS Collaboration, Search for dark matter in events with heavy quarks and missing transverse momentum in $\mathrm{pp}$ collisions with the ATLAS detector, Eur. Phys. J. C 75, 92 (2015).

[17] CMS Collaboration, Search for dark matter produced in association with heavy-flavor quark pairs in proton-proton collisions at $\sqrt{s}=13 \mathrm{TeV}$, Eur. Phys. J. C 77, 845 (2017).

[18] CMS Collaboration, The CMS trigger system, J. Instrum. 12, P01020 (2017). 
[19] CMS Collaboration, The CMS experiment at the CERN LHC, J. Instrum. 3, S08004 (2008).

[20] CMS Collaboration, Particle-flow reconstruction and global event description with the CMS detector, J. Instrum. 12, P10003 (2017).

[21] M. Cacciari, G. P. Salam, and G. Soyez, The anti- $k_{T}$ jet clustering algorithm, J. High Energy Phys. 04 (2008) 063.

[22] M. Cacciari, G. P. Salam, and G. Soyez, FastJet user manual, Eur. Phys. J. C 72, 1896 (2012).

[23] CMS Collaboration, Determination of jet energy calibration and transverse momentum resolution in CMS, J. Instrum. 6, P11002 (2011).

[24] M. Cacciari, G. P. Salam, and G. Soyez, The catchment area of jets, J. High Energy Phys. 04 (2008) 005.

[25] CMS Collaboration, Jet algorithms performance in $13 \mathrm{TeV}$ data, CMS physics analysis summary, CERN Report No. CMS-PAS-JME-16-003, 2017, https://cds.cern.ch/ record/2256875.

[26] CMS Collaboration, Identification of heavy-flavour jets with the CMS detector in pp collisions at $13 \mathrm{TeV}$, J. Instrum. 13, P05011 (2018).

[27] CMS Collaboration, Performance of electron reconstruction and selection with the CMS detector in proton-proton collisions at $\sqrt{s}=8 \mathrm{TeV}$, J. Instrum. 10, P06005 (2015).

[28] P. Nason, A new method for combining NLO QCD with shower Monte Carlo algorithms, J. High Energy Phys. 11 (2004) 040.

[29] S. Frixione, P. Nason, and C. Oleari, Matching NLO QCD computations with parton shower simulations: The POWHEG method, J. High Energy Phys. 11 (2007) 070.

[30] S. Alioli, P. Nason, C. Oleari, and E. Re, A general framework for implementing NLO calculations in shower Monte Carlo programs: The POWHEG BOX, J. High Energy Phys. 06 (2010) 043.

[31] C. Oleari, The POWHEG-BOX, Nucl. Phys. B, Proc. Suppl. 205-206, 36 (2010).

[32] J. Alwall, R. Frederix, S. Frixione, V. Hirschi, F. Maltoni, O. Mattelaer, H. S. Shao, T. Stelzer, P. Torrielli, and M. Zaro, The automated computation of tree-level and next-toleading order differential cross sections, and their matching to parton shower simulations, J. High Energy Phys. 07 (2014) 079.

[33] A. Denner, S. Dittmaier, T. Kasprzik, and A. Muck, Electroweak corrections to $W+$ jet hadroproduction including leptonic W-boson decays, J. High Energy Phys. 08 (2009) 075.

[34] A. Denner, S. Dittmaier, T. Kasprzik, and A. Muck, Electroweak corrections to dilepton + jet production at hadron colliders, J. High Energy Phys. 06 (2011) 069.

[35] A. Denner, S. Dittmaier, T. Kasprzik, and A. Maeck, Electroweak corrections to monojet production at the Tevatron and the LHC, Eur. Phys. J. C 73, 2297 (2013).

[36] J. H. Kuhn, A. Kulesza, S. Pozzorini, and M. Schulze, Electroweak corrections to hadronic photon production at large transverse momenta, J. High Energy Phys. 03 (2006) 059 .

[37] S. Kallweit, J. M. Lindert, P. Maierhafer, S. Pozzorini, and M. Schanherr, NLO electroweak automation and precise predictions for $W+$ multijet production at the LHC, J. High Energy Phys. 04 (2015) 012.
[38] S. Kallweit, J. M. Lindert, P. Maierhofer, S. Pozzorini, and M. Schonherr, NLO QCD + EW predictions for $V+$ jets including off-shell vector-boson decays and multijet merging, J. High Energy Phys. 04 (2016) 021.

[39] R. D. Ball et al. (NNPDF Collaboration), Parton distributions for the LHC Run II, J. High Energy Phys. 04 (2015) 040 .

[40] T. Sjöstrand, S. Mrenna, and P.Z. Skands, A brief introduction to PYTHIA 8.1, Comput. Phys. Commun. 178, 852 (2008).

[41] CMS Collaboration, Event generator tunes obtained from underlying event and multiparton scattering measurements, Eur. Phys. J. C 76, 155 (2016).

[42] S. Agostinelli et al. (GEANT4 Collaboration), GEANT4-A simulation toolkit, Nucl. Instrum. Methods Phys. Res., Sect. A 506, 250 (2003).

[43] D. Abercrombie et al., Dark matter benchmark models for early LHC Run-2 searches: Report of the ATLAS/CMS dark matter forum, arXiv:1507.00966.

[44] A. Albert et al., Towards the next generation of simplified dark matter models, Phys. Dark Universe 16, 49 (2017).

[45] P. Harris, V. V. Khoze, M. Spannowsky, and C. Williams, Constraining dark sectors at colliders: Beyond the effective theory approach, Phys. Rev. D 91, 055009 (2015).

[46] Y. Bai, H.-C. Cheng, J. Gallicchio, and J. Gu, Stop the top background of the stop search, J. High Energy Phys. 07 (2012) 110.

[47] C. G. Lester and D. J. Summers, Measuring masses of semiinvisibly decaying particles pair produced at hadron colliders, Phys. Lett. B 463, 99 (1999).

[48] M. Burns, K. Kong, K. T. Matchev, and M. Park, Using subsystem $M_{T 2}$ for complete mass determinations in decay chains with missing energy at hadron colliders, J. High Energy Phys. 03 (2009) 143.

[49] H.-C. Cheng and Z. Han, Minimal kinematic constraints and $m_{T 2}$, J. High Energy Phys. 12 (2008) 063.

[50] C. Patrignani et al. (Particle Data Group), Review of particle physics, Chin. Phys. C 40, 100001 (2016).

[51] L. Moneta, K. Belasco, K. S. Cranmer, S. Kreiss, A. Lazzaro, D. Piparo, G. Schott, W. Verkerke, and M. Wolf, The RooStats Project, Proc. Sci., ACAT2010 (2010) 057 [https://pos.sissa.it/093/057].

[52] CMS Collaboration, CMS luminosity measurements for the 2016 data taking period, CMS Physics Analysis Summary, CERN Report No. CMS-PAS-LUM-17-001, 2017, https:// cds.cern.ch/record/2257069.

[53] V. Khachatryan et al. (CMS Collaboration), Measurement of differential cross sections for top quark pair production using the lepton + jets final state in proton-proton collisions at $13 \mathrm{TeV}$, Phys. Rev. D 95, 092001 (2017).

[54] T. Junk, Confidence level computation for combining searches with small statistics, Nucl. Instrum. Methods Phys. Res., Sect. A 434, 435 (1999).

[55] A. L. Read, Presentation of search results: The $C L_{s}$ technique, J. Phys. G 28, 2693 (2002).

[56] G. Cowan, K. Cranmer, E. Gross, and O. Vitells, Asymptotic formulae for likelihood-based tests of new physics, Eur. Phys. J. C 71, 1554 (2011); Erratum, Eur. Phys. J. C 73, 2501 (2011). 
[57] CMS Collaboration, Search for top squarks and dark matter particles in opposite-charge dilepton final states at $\sqrt{s}=13$ TeV, Phys. Rev. D 97, 032009 (2018).

[58] ATLAS Collaboration, Search for top-squark pair production in final states with one lepton, jets, and missing transverse momentum using $36.1 \mathrm{fb}^{-1}$ of $\sqrt{s}=13 \mathrm{TeV}$ pp collision data with the ATLAS detector, J. High Energy Phys. 06 (2018) 108.

[59] ATLAS Collaboration, Search for dark matter produced in association with bottom or top quarks in $\sqrt{s}=13 \mathrm{TeV}$ pp collisions with the ATLAS detector, Eur. Phys. J. C 78, 18 (2018).

A. M. Sirunyan, ${ }^{1}$ A. Tumasyan, ${ }^{1}$ W. Adam, ${ }^{2}$ F. Ambrogi, ${ }^{2}$ E. Asilar, ${ }^{2}$ T. Bergauer, ${ }^{2}$ J. Brandstetter, ${ }^{2}$ M. Dragicevic, ${ }^{2}$ J. Erö, ${ }^{2}$ A. Escalante Del Valle, ${ }^{2}$ M. Flechl, ${ }^{2}$ R. Frühwirth, ${ }^{2, b}$ V. M. Ghete, ${ }^{2}$ J. Hrubec, ${ }^{2}$ M. Jeitler, ${ }^{2, b}$ N. Krammer, ${ }^{2}$ I. Krätschmer, ${ }^{2}$ D. Liko, ${ }^{2}$ T. Madlener, ${ }^{2}$ I. Mikulec, ${ }^{2}$ N. Rad, ${ }^{2}$ H. Rohringer, ${ }^{2}$ J. Schieck, ${ }^{2, b}$ R. Schöfbeck, ${ }^{2}$ M. Spanring, ${ }^{2}$ D. Spitzbart, ${ }^{2}$ A. Taurok, ${ }^{2}$ W. Waltenberger, ${ }^{2}$ J. Wittmann, ${ }^{2}$ C.-E. Wulz, ${ }^{2, b}$ M. Zarucki, ${ }^{2}$ V. Chekhovsky, ${ }^{3}$ V. Mossolov, ${ }^{3}$

J. Suarez Gonzalez, ${ }^{3}$ E. A. De Wolf, ${ }^{4}$ D. Di Croce, ${ }^{4}$ X. Janssen, ${ }^{4}$ J. Lauwers, ${ }^{4}$ M. Pieters, ${ }^{4}$ M. Van De Klundert, ${ }^{4}$ H. Van Haevermaet, ${ }^{4}$ P. Van Mechelen, ${ }^{4}$ N. Van Remortel, ${ }^{4}$ S. Abu Zeid, ${ }^{5}$ F. Blekman, ${ }^{5}$ J. D'Hondt, ${ }^{5}$ I. De Bruyn, ${ }^{5}$ J. De Clercq, ${ }^{5}$ K. Deroover, ${ }^{5}$ G. Flouris, ${ }^{5}$ D. Lontkovskyi, ${ }^{5}$ S. Lowette, ${ }^{5}$ I. Marchesini ${ }^{5}$ S. Moortgat, ${ }^{5}$ L. Moreels, ${ }^{5}$ Q. Python, ${ }^{5}$ K. Skovpen, ${ }^{5}$ S. Tavernier, ${ }^{5}$ W. Van Doninck, ${ }^{5}$ P. Van Mulders, ${ }^{5}$ I. Van Parijs, ${ }^{5}$ D. Beghin, ${ }^{6}$ B. Bilin, ${ }^{6}$ H. Brun, ${ }^{6}$ B. Clerbaux, ${ }^{6}$ G. De Lentdecker, ${ }^{6}$ H. Delannoy, ${ }_{6}^{6}$ B. Dorney, ${ }^{6}$ G. Fasanella, ${ }^{6}$ L. Favart, ${ }^{6}$ R. Goldouzian, ${ }^{6}$ A. Grebenyuk, ${ }^{6}$ A. K. Kalsi, ${ }^{6}$ T. Lenzi, ${ }^{6}$ J. Luetic, ${ }^{6}$ N. Postiau, ${ }^{6}$ E. Starling, ${ }^{6}$ L. Thomas, ${ }^{6}$ C. Vander Velde, ${ }^{6}$ P. Vanlaer, ${ }^{6}$ D. Vannerom, ${ }^{6}$ Q. Wang, ${ }^{6}$ T. Cornelis, ${ }^{7}$ D. Dobur, ${ }^{7}$ A. Fagot, ${ }^{7}$ M. Gul, ${ }^{7}$ I. Khvastunov,${ }^{7, c}$ D. Poyraz, ${ }^{7}$ C. Roskas, ${ }^{7}$ D. Trocino, ${ }^{7}$ M. Tytgat, ${ }^{7}$ W. Verbeke, ${ }^{7}$ B. Vermassen, ${ }^{7}$ M. Vit, ${ }^{7}$ N. Zaganidis, ${ }^{7}$ H. Bakhshiansohi, ${ }^{8}$ O. Bondu, ${ }^{8}$ S. Brochet, ${ }^{8}$ G. Bruno,${ }^{8}$ C. Caputo, ${ }^{8}$ P. David, ${ }^{8}$ C. Delaere, ${ }^{8}$ M. Delcourt, ${ }^{8}$ B. Francois, ${ }^{8}$ A. Giammanco, ${ }^{8}$ G. Krintiras, ${ }^{8}$ V. Lemaitre, ${ }^{8}$ A. Magitteri, ${ }^{8}$ A. Mertens, ${ }^{8}$ M. Musich, ${ }^{8}$ K. Piotrzkowski, ${ }^{8}$ A. Saggio, ${ }^{8}$ M. Vidal Marono, ${ }^{8}$ S. Wertz, ${ }^{8}$ J. Zobec, ${ }^{8}$ F. L. Alves, ${ }^{9}$ G. A. Alves, ${ }^{9}$ M. Correa Martins Junior, ${ }^{9}$ G. Correia Silva, ${ }^{9}$ C. Hensel, ${ }^{9}$ A. Moraes, ${ }^{9}$ M. E. Pol, ${ }^{9}$ P. Rebello Teles, ${ }^{9}$

E. Belchior Batista Das Chagas, ${ }^{10}$ W. Carvalho, ${ }^{10}$ J. Chinellato, ${ }^{10, \mathrm{~d}}$ E. Coelho, ${ }^{10}$ E. M. Da Costa, ${ }^{10}$ G. G. Da Silveira, ${ }^{10, \mathrm{e}}$ D. De Jesus Damiao, ${ }^{10}$ C. De Oliveira Martins, ${ }^{10}$ S. Fonseca De Souza ${ }^{10}$ H. Malbouisson, ${ }^{10}$ D. Matos Figueiredo, ${ }^{10}$ M. Melo De Almeida, ${ }^{10}$ C. Mora Herrera, ${ }^{10}$ L. Mundim, ${ }^{10}$ H. Nogima, ${ }^{10}$ W. L. Prado Da Silva, ${ }^{10}$ L. J. Sanchez Rosas,${ }^{10}$ A. Santoro, ${ }^{10}$ A. Sznajder, ${ }^{10}$ M. Thiel, ${ }^{10}$ E. J. Tonelli Manganote, ${ }^{10, \mathrm{~d}}$ F. Torres Da Silva De Araujo, ${ }^{10}$ A. Vilela Pereira, ${ }^{10}$ S. Ahuja, ${ }^{11 \mathrm{a}}$ C. A. Bernardes,${ }^{11 \mathrm{a}}$ L. Calligaris, ${ }^{11 \mathrm{a}}$ T. R. Fernandez Perez Tomei, ${ }^{11 \mathrm{a}}$ E. M. Gregores, ${ }^{11 \mathrm{a}, 1 \mathrm{~b}}$

P. G. Mercadante, ${ }^{11 \mathrm{a}, 11 \mathrm{~b}}$ S. F. Novaes, ${ }^{11 \mathrm{a}}$ Sandra S. Padula, ${ }^{11 \mathrm{a}}$ D. Romero Abad, ${ }^{11 \mathrm{a}, 1 \mathrm{~b}}$ A. Aleksandrov, ${ }^{12}$ R. Hadjiiska, ${ }^{12}$ P. Iaydjiev, ${ }^{12}$ A. Marinov, ${ }^{12}$ M. Misheva, ${ }^{12}$ M. Rodozov, ${ }^{12}$ M. Shopova, ${ }^{12}$ G. Sultanov ${ }^{12}$ A. Dimitrov, ${ }^{13}$ L. Litov, ${ }^{13}$ B. Pavlov ${ }^{13}$ P. Petkov, ${ }^{13}$ W. Fang, ${ }^{14, f}$ X. Gao,${ }^{14, f}$ L. Yuan, ${ }^{14}$ M. Ahmad, ${ }^{15}$ J. G. Bian, ${ }^{15}$ G. M. Chen, ${ }^{15}$ H. S. Chen, ${ }^{15}$ M. Chen, ${ }^{15}$ Y. Chen, ${ }^{15}$ C. H. Jiang, ${ }^{15}$ D. Leggat,${ }^{15}$ H. Liao, ${ }^{15}$ Z. Liu, ${ }^{15}$ F. Romeo, ${ }^{15}$ S. M. Shaheen, ${ }^{15, g}$ A. Spiezia, ${ }^{15}$ J. Tao, ${ }^{15}$ C. Wang, ${ }^{15}$ Z. Wang, ${ }^{15}$ E. Yazgan, ${ }^{15}$ H. Zhang, ${ }^{15}$ J. Zhao, ${ }^{15}$ Y. Ban, ${ }^{16}$ G. Chen, ${ }^{16}$ A. Levin, ${ }^{16}$ J. Li,${ }^{16}$ L. Li, ${ }^{16}$ Q. Li ${ }^{16}$ Y. Mao, ${ }^{16}$ S. J. Qian, ${ }^{16}$ D. Wang, ${ }^{16}$ Z. Xu, ${ }^{16}$ Y. Wang, ${ }^{17}$ C. Avila, ${ }^{18}$ A. Cabrera, ${ }^{18}$ C. A. Carrillo Montoya, ${ }^{18}$ L. F. Chaparro Sierra, ${ }^{18}$ C. Florez, ${ }^{18}$ C. F. González Hernández,${ }^{18}$ M. A. Segura Delgado, ${ }^{18}$ B. Courbon, ${ }^{19}$ N. Godinovic, ${ }^{19}$ D. Lelas, ${ }^{19}$ I. Puljak, ${ }^{19}$ T. Sculac, ${ }^{19}$ Z. Antunovic, ${ }^{20}$ M. Kovac, ${ }^{20}$ V. Brigljevic, ${ }^{21}$ D. Ferencek, ${ }^{21}$ K. Kadija, ${ }^{21}$ B. Mesic, ${ }^{21}$ A. Starodumov, ${ }^{21, h}$ T. Susa, ${ }^{21}$ M. W. Ather, ${ }^{22}$ A. Attikis, ${ }^{22}$ M. Kolosova, ${ }^{22}$ G. Mavromanolakis, ${ }^{22}$ J. Mousa, ${ }^{22}$ C. Nicolaou, ${ }^{22}$ F. Ptochos, ${ }^{22}$ P. A. Razis, ${ }^{22}$ H. Rykaczewski, ${ }^{22}$ M. Finger ${ }^{23, i}$ M. Finger Jr., ${ }^{23, i}$ E. Ayala, ${ }^{24}$ E. Carrera Jarrin, ${ }^{25}$ Y. Assran, ${ }^{26, j, k}$ S. Elgammal, ${ }^{26, j}$ Y. Mohammed, ${ }^{26,1}$ S. Bhowmik, ${ }^{27}$ A. Carvalho Antunes De Oliveira, ${ }^{27}$ R. K. Dewanjee, ${ }^{27}$ K. Ehataht, ${ }^{27}$ M. Kadastik, ${ }^{27}$ M. Raidal,${ }^{27}$ C. Veelken, ${ }^{27}$ P. Eerola, ${ }^{28}$ H. Kirschenmann, ${ }^{28}$ J. Pekkanen, ${ }^{28}$ M. Voutilainen, ${ }^{28}$

J. Havukainen, ${ }^{29}$ J. K. Heikkilä, ${ }^{29}$ T. Järvinen, ${ }^{29}$ V. Karimäki, ${ }^{29}$ R. Kinnunen, ${ }^{29}$ T. Lampén, ${ }^{29}$ K. Lassila-Perini, ${ }^{29}$ S. Laurila, ${ }^{29}$ S. Lehti, ${ }^{29}$ T. Lindén, ${ }^{29}$ P. Luukka, ${ }^{29}$ T. Mäenpää, ${ }^{29}$ H. Siikonen, ${ }^{29}$ E. Tuominen, ${ }^{29}$ J. Tuominiemi, ${ }^{29}$ T. Tuuva,${ }^{30}$ M. Besancon, ${ }^{31}$ F. Couderc, ${ }^{31}$ M. Dejardin, ${ }^{31}$ D. Denegri, ${ }^{31}$ J. L. Faure, ${ }^{31}$ F. Ferri, ${ }^{31}$ S. Ganjour, ${ }^{31}$ A. Givernaud,${ }^{31}$ P. Gras, ${ }^{31}$ G. Hamel de Monchenault, ${ }^{31}$ P. Jarry, ${ }^{31}$ C. Leloup, ${ }^{31}$ E. Locci, ${ }^{31}$ J. Malcles, ${ }^{31}$ G. Negro, ${ }^{31}$ J. Rander, ${ }^{31}$ A. Rosowsky, ${ }^{31}$ M. Ö. Sahin, ${ }^{31}$ M. Titov, ${ }^{31}$ A. Abdulsalam, ${ }^{32, \mathrm{~m}}$ C. Amendola, ${ }^{32}$ I. Antropov,${ }^{32}$ F. Beaudette, ${ }^{32}$ P. Busson, ${ }^{32}$ C. Charlot,${ }^{32}$ R. Granier de Cassagnac, ${ }^{32}$ I. Kucher, ${ }^{32}$ A. Lobanov, ${ }^{32}$ J. Martin Blanco, ${ }^{32}$ M. Nguyen, ${ }^{32}$ C. Ochando, ${ }^{32}$ G. Ortona ${ }^{32}$ P. Paganini, ${ }^{32}$ P. Pigard, ${ }^{32}$ R. Salerno, ${ }^{32}$ J. B. Sauvan, ${ }^{32}$ Y. Sirois, ${ }^{32}$ A. G. Stahl Leiton, ${ }^{32}$ A. Zabi, ${ }^{32}$ A. Zghiche, ${ }^{32}$ J.-L. Agram, ${ }^{33, n}$ J. Andrea ${ }^{33}$ D. Bloch, ${ }^{33}$ J.-M. Brom, ${ }^{33}$ E. C. Chabert, ${ }^{33}$ V. Cherepanov, ${ }^{33}$ C. Collard ${ }^{33}$ E. Conte,,${ }^{33, n}$ J.-C. Fontaine, ${ }^{33, n}$ D. Gelé, ${ }^{33}$ U. Goerlach, ${ }^{33}$ M. Jansová, ${ }^{33}$ A.-C. Le Bihan, ${ }^{33}$ N. Tonon, ${ }^{33}$ P. Van Hove, ${ }^{33}$ S. Gadrat, ${ }^{34}$ 
S. Beauceron, ${ }^{35}$ C. Bernet, ${ }^{35}$ G. Boudoul,${ }^{35}$ N. Chanon, ${ }^{35}$ R. Chierici,${ }^{35}$ D. Contardo,${ }^{35}$ P. Depasse,${ }^{35}$ H. El Mamouni, ${ }^{35}$ J. Fay, ${ }^{35}$ L. Finco, ${ }^{35}$ S. Gascon, ${ }^{35}$ M. Gouzevitch,${ }^{35}$ G. Grenier, ${ }^{35}$ B. Ille, ${ }^{35}$ F. Lagarde,${ }^{35}$ I. B. Laktineh, ${ }^{35}$ H. Lattaud,${ }^{35}$ M. Lethuillier, ${ }^{35}$ L. Mirabito, ${ }^{35}$ A. L. Pequegnot, ${ }^{35}$ S. Perries, ${ }^{35}$ A. Popov, ${ }^{35,0}$ V. Sordini, ${ }^{35}$ M. Vander Donckt,,${ }^{35}$ S. Viret,${ }^{35}$ S. Zhang, ${ }^{35}$ A. Khvedelidze, ${ }^{36, i}$ Z. Tsamalaidze, ${ }^{37, i}$ C. Autermann, ${ }^{38}$ L. Feld ${ }^{38}$ M. K. Kiesel,${ }^{38}$ K. Klein,${ }^{38}$ M. Lipinski, ${ }^{38}$ M. Preuten,${ }^{38}$ M. P. Rauch ${ }^{38}$ C. Schomakers, ${ }^{38}$ J. Schulz,${ }^{38}$ M. Teroerde, ${ }^{38}$ B. Wittmer, ${ }^{38}$ V. Zhukov,${ }^{38,0}$ A. Albert, ${ }^{39}$ D. Duchardt ${ }^{39}$ M. Endres ${ }^{39}$ M. Erdmann, ${ }^{39}$ T. Esch, ${ }^{39}$ R. Fischer, ${ }^{39}$ S. Ghosh, ${ }^{39}$ A. Güth, ${ }^{39}$ T. Hebbeker, ${ }^{39}$ C. Heidemann, ${ }^{39}$ K. Hoepfner, ${ }^{39}$ H. Keller, ${ }^{39}$ S. Knutzen, ${ }^{39}$ L. Mastrolorenzo, ${ }^{39}$ M. Merschmeyer, ${ }^{39}$ A. Meyer, ${ }^{39}$ P. Millet, ${ }^{39}$ S. Mukherjee, ${ }^{39}$ T. Pook, ${ }^{39}$ M. Radziej, ${ }^{39}$ H. Reithler, ${ }^{39}$ M. Rieger,${ }^{39}$ F. Scheuch, ${ }^{39}$ A. Schmidt, ${ }^{39}$ D. Teyssier, ${ }^{39}$ G. Flügge, ${ }^{40}$ O. Hlushchenko, ${ }^{40}$ T. Kress, ${ }^{40}$ A. Künsken, ${ }^{40}$ T. Müller, ${ }^{40}$ A. Nehrkorn, ${ }^{40}$ A. Nowack, ${ }^{40}$ C. Pistone, ${ }^{40}$ O. Pooth, ${ }^{40}$ D. Roy, ${ }^{40}$ H. Sert, ${ }^{40}$ A. Stahl, ${ }^{40, p}$ M. Aldaya Martin, ${ }^{41}$ T. Arndt,${ }^{41}$ C. Asawatangtrakuldee, ${ }^{41}$ I. Babounikau, ${ }^{41}$ K. Beernaert,${ }^{41}$ O. Behnke, ${ }^{41}$ U. Behrens, ${ }^{41}$ A. Bermúdez Martínez, ${ }^{41}$ D. Bertsche, ${ }^{41}$ A. A. Bin Anuar, ${ }^{41}$ K. Borras, ${ }^{41, q}$ V. Botta, ${ }^{41}$ A. Campbell, ${ }^{41}$ P. Connor, ${ }^{41}$ C. Contreras-Campana, ${ }^{41}$ F. Costanza, ${ }^{41}$ V. Danilov,${ }^{41}$ A. De Wit ${ }^{41}$ M. M. Defranchis, ${ }^{41}$ C. Diez Pardos, ${ }^{41}$ D. Domínguez Damiani, ${ }^{41}$ G. Eckerlin, ${ }^{41}$ T. Eichhorn, ${ }^{41}$ A. Elwood, ${ }^{41}$ E. Eren, ${ }^{41}$ E. Gallo, ${ }^{41, r}$ A. Geiser,${ }^{41}$ J. M. Grados Luyando, ${ }^{41}$ A. Grohsjean, ${ }^{41}$ P. Gunnellini, ${ }^{41}$ M. Guthoff, ${ }^{41}$ M. Haranko, ${ }^{41}$ A. Harb,${ }^{41}$ J. Hauk, ${ }^{41}$ H. Jung, ${ }^{41}$ M. Kasemann, ${ }^{41}$ J. Keaveney, ${ }^{41}$ C. Kleinwort, ${ }^{41}$ J. Knolle, ${ }^{41}$ D. Krücker, ${ }^{41}$ W. Lange, ${ }^{41}$ A. Lelek, ${ }^{41}$ T. Lenz, ${ }^{41}$ K. Lipka, ${ }^{41}$ W. Lohmann, ${ }^{41, \mathrm{~s}}$ R. Mankel, ${ }^{41}$ I.-A. Melzer-Pellmann, ${ }^{41}$ A. B. Meyer, ${ }^{41}$ M. Meyer, ${ }^{41}$ M. Missiroli, ${ }^{41}$ G. Mittag, ${ }^{41}$ J. Mnich, ${ }^{41}$ V. Myronenko, ${ }^{41}$ S. K. Pflitsch,${ }^{41}$ D. Pitzl,${ }^{41}$ A. Raspereza,${ }^{41}$ M. Savitskyi,${ }^{41}$ P. Saxena, ${ }^{41}$ P. Schütze,${ }^{41}$ C. Schwanenberger ${ }^{41}$ R. Shevchenko, ${ }^{41}$ A. Singh, ${ }^{41}$ H. Tholen, ${ }^{41}$ O. Turkot, ${ }^{41}$ A. Vagnerini, ${ }^{41}$ G. P. Van Onsem, ${ }^{41}$ R. Walsh, ${ }^{41}$ Y. Wen, ${ }^{41}$

K. Wichmann, ${ }^{41}$ C. Wissing, ${ }^{41}$ O. Zenaiev, ${ }^{41}$ R. Aggleton, ${ }^{42}$ S. Bein, ${ }^{42}$ L. Benato ${ }^{42}$ A. Benecke, ${ }^{42}$ V. Blobel, ${ }^{42}$ M. Centis Vignali, ${ }^{42}$ T. Dreyer, ${ }^{42}$ E. Garutti, ${ }^{42}$ D. Gonzalez, ${ }^{42}$ J. Haller, ${ }^{42}$ A. Hinzmann, ${ }^{42}$ A. Karavdina, ${ }^{42}$ G. Kasieczka, ${ }^{42}$ R. Klanner, ${ }^{42}$ R. Kogler, ${ }^{42}$ N. Kovalchuk ${ }^{42}$ S. Kurz, ${ }^{42}$ V. Kutzner, ${ }^{42}$ J. Lange, ${ }^{42}$ D. Marconi, ${ }^{42}$ J. Multhaup, ${ }^{42}$ M. Niedziela, ${ }^{42}$ D. Nowatschin, ${ }^{42}$ A. Perieanu, ${ }^{42}$ A. Reimers, ${ }^{42}$ O. Rieger, ${ }^{42}$ C. Scharf, ${ }^{42}$ P. Schleper, ${ }^{42}$ S. Schumann, ${ }^{42}$ J. Schwandt, ${ }^{42}$ J. Sonneveld, ${ }^{42}$ H. Stadie, ${ }^{42}$ G. Steinbrück, ${ }^{42}$ F. M. Stober, ${ }^{42}$ M. Stöver, ${ }^{42}$ D. Troendle, ${ }^{42}$ A. Vanhoefer, ${ }^{42}$ B. Vormwald, ${ }^{42}$ M. Akbiyik, ${ }^{43}$ C. Barth, ${ }^{43}$ M. Baselga, ${ }^{43}$ S. Baur, ${ }^{43}$ E. Butz, ${ }^{43}$ R. Caspart ${ }^{43}$ T. Chwalek, ${ }^{43}$ F. Colombo, ${ }^{43}$ W. De Boer,${ }^{43}$ A. Dierlamm, ${ }^{43}$ K. El Morabit, ${ }^{43}$ N. Faltermann, ${ }^{43}$ B. Freund, ${ }^{43}$ M. Giffels, ${ }^{43}$ M. A. Harrendorf, ${ }^{43}$ F. Hartmann, ${ }^{43, p}$ S. M. Heindl, ${ }^{43}$ U. Husemann, ${ }^{43}$ F. Kassel, ${ }^{43, p}$ I. Katkov, ${ }^{43, o}$ S. Kudella, ${ }^{43}$ H. Mildner, ${ }^{43}$ S. Mitra, ${ }^{43}$ M. U. Mozer, ${ }^{43}$ Th. Müller, ${ }^{43}$ M. Plagge, ${ }^{43}$ G. Quast, ${ }^{43}$ K. Rabbertz, ${ }^{43}$ M. Schröder, ${ }^{43}$ I. Shvetsov,${ }^{43}$ G. Sieber, ${ }^{43}$ H. J. Simonis ${ }^{43}$ R. Ulrich,${ }^{43}$ S. Wayand, ${ }^{43}$ M. Weber, ${ }^{43}$ T. Weiler ${ }^{43}$ S. Williamson, ${ }^{43}$ C. Wöhrmann, ${ }^{43}$ R. Wolf, ${ }^{43}$ G. Anagnostou, ${ }^{44}$ G. Daskalakis, ${ }^{44}$ T. Geralis, ${ }^{44}$ A. Kyriakis, ${ }^{44}$ D. Loukas,${ }^{44}$ G. Paspalaki, ${ }^{44}$ I. Topsis-Giotis, ${ }^{44}$ G. Karathanasis, ${ }^{45}$ S. Kesisoglou, ${ }^{45}$ P. Kontaxakis, ${ }^{45}$ A. Panagiotou ${ }^{45}$ I. Papavergou, ${ }^{45}$ N. Saoulidou, ${ }^{45}$ E. Tziaferi, ${ }^{45}$ K. Vellidis, ${ }^{45}$ K. Kousouris, ${ }^{46}$ I. Papakrivopoulos, ${ }^{46}$ G. Tsipolitis, ${ }^{46}$ I. Evangelou, ${ }^{47}$ C. Foudas, ${ }^{47}$ P. Gianneios, ${ }^{47}$ P. Katsoulis,${ }^{47}$ P. Kokkas,${ }^{47}$ S. Mallios,${ }^{47}$ N. Manthos, ${ }^{47}$ I. Papadopoulos, ${ }^{47}$ E. Paradas, ${ }^{47}$ J. Strologas, ${ }^{47}$ F. A. Triantis,${ }^{47}$ D. Tsitsonis, ${ }^{47}$ M. Bartók,${ }^{48, t}$ M. Csanad ${ }^{48}$ N. Filipovic, ${ }^{48}$ P. Major, ${ }^{48}$ M. I. Nagy,${ }^{48}$ G. Pasztor, ${ }^{48}$ O. Surányi,${ }^{48}$ G. I. Veres,${ }^{48}$ G. Bencze, ${ }^{49}$ C. Hajdu,${ }^{49}$ D. Horvath, ${ }^{49, u}$ Á. Hunyadi, ${ }^{49}$ F. Sikler, ${ }^{49}$ T. Á. Vámi ${ }^{49}$ V. Veszpremi, ${ }^{49}$ G. Vesztergombi, ${ }^{49, a, t}$ N. Beni, ${ }^{50}$ S. Czellar, ${ }^{50}$ J. Karancsi, ${ }^{50, v}$ A. Makovec, ${ }^{50}$ J. Molnar, ${ }^{50}$ Z. Szillasi,${ }^{50}$ P. Raics,${ }^{51}$ Z. L. Trocsanyi,${ }^{51}$ B. Ujvari, ${ }^{51}$ S. Choudhury, ${ }^{52}$ J. R. Komaragiri, ${ }^{52}$ P. C. Tiwari, ${ }^{52}$ S. Bahinipati, ${ }^{53, w}$ C. Kar, ${ }^{53}$ P. Mal, ${ }^{53}$ K. Mandal, ${ }^{53}$ A. Nayak,${ }^{53, \mathrm{x}}$ D. K. Sahoo, ${ }^{53, w}$ S. K. Swain, ${ }^{53}$ S. Bansal, ${ }^{54}$ S. B. Beri, ${ }^{54}$ V. Bhatnagar, ${ }^{54}$ S. Chauhan,${ }^{54}$ R. Chawla,${ }^{54}$ N. Dhingra, ${ }^{54}$ R. Gupta, ${ }^{54}$ A. Kaur, ${ }^{54}$ A. Kaur, ${ }^{54}$ M. Kaur, ${ }^{54}$ S. Kaur, ${ }^{54}$ R. Kumar, ${ }^{54}$ P. Kumari, ${ }^{54}$ M. Lohan,${ }^{54}$ A. Mehta ${ }^{54}$ K. Sandeep, ${ }^{54}$ S. Sharma, ${ }^{54}$ J. B. Singh, ${ }^{54}$ G. Walia, ${ }^{54}$ A. Bhardwaj, ${ }^{55}$ B. C. Choudhary, ${ }^{55}$ R. B. Garg, ${ }^{55}$ M. Gola,${ }^{55}$ S. Keshri, ${ }^{55}$ Ashok Kumar, ${ }^{55}$ S. Malhotra, ${ }^{55}$ M. Naimuddin,${ }^{55}$ P. Priyanka ${ }^{55}$ K. Ranjan, ${ }^{55}$ Aashaq Shah,${ }^{55}$ R. Sharma,${ }^{55}$ R. Bhardwaj,${ }^{56, y}$ M. Bharti, ${ }^{56}$ R. Bhattacharya,${ }^{56}$ S. Bhattacharya ${ }^{56}$ U. Bhawandeep, ${ }^{56, y}$ D. Bhowmik, ${ }^{56}$ S. Dey, ${ }^{56}$ S. Dutt, ${ }^{56, y}$ S. Dutta ${ }^{56}$ S. Ghosh,${ }^{56}$ K. Mondal, ${ }^{56}$ S. Nandan, ${ }^{56}$ A. Purohit,${ }^{56}$ P. K. Rout,${ }^{56}$ A. Roy, ${ }^{56}$ S. Roy Chowdhury, ${ }^{56}$ G. Saha,${ }^{56}$ S. Sarkar, ${ }^{56}$ M. Sharan, ${ }^{56}$ B. Singh,${ }^{56}$ S. Thakur,${ }^{56, y}$ P. K. Behera, ${ }^{57}$ R. Chudasama ${ }^{58}$ D. Dutta, ${ }^{58}$ V. Jha ${ }^{58}$ V. Kumar, ${ }^{58}$ P. K. Netrakanti, ${ }^{58}$ L. M. Pant,${ }^{58}$ P. Shukla, ${ }^{58}$ T. Aziz, ${ }^{59}$ M. A. Bhat ${ }^{59}$

S. Dugad, ${ }^{59}$ G. B. Mohanty, ${ }^{59}$ N. Sur, ${ }^{59}$ B. Sutar, ${ }^{59}$ Ravindra Kumar Verma, ${ }^{59}$ S. Banerjee, ${ }^{60}$ S. Bhattacharya,${ }^{60}$ S. Chatterjee, ${ }^{60}$ P. Das, ${ }^{60}$ M. Guchait ${ }^{60}$ Sa. Jain, ${ }^{60}$ S. Karmakar, ${ }^{60}$ S. Kumar, ${ }^{60}$ M. Maity, ${ }^{60, z}$ G. Majumder, ${ }^{60}$ K. Mazumdar,${ }^{60}$ N. Sahoo, ${ }^{60}$ T. Sarkar ${ }^{60, z}$ S. Chauhan, ${ }^{61}$ S. Dube, ${ }^{61}$ V. Hegde, ${ }^{61}$ A. Kapoor, ${ }^{61}$ K. Kothekar, ${ }^{61}$ S. Pandey,${ }^{61}$ A. Rane, ${ }^{61}$ S. Sharma, ${ }^{61}$ S. Chenarani, ${ }^{62, a a}$ E. Eskandari Tadavani, ${ }^{62}$ S. M. Etesami, ${ }^{62, \text { aa }}$ M. Khakzad ${ }^{62}$ M. Mohammadi Najafabadi, ${ }^{62}$ M. Naseri, ${ }^{62}$ F. Rezaei Hosseinabadi ${ }^{62}$ B. Safarzadeh,${ }^{62, b b}$ M. Zeinali, ${ }^{62}$ M. Felcini, ${ }^{63}$ M. Grunewald, ${ }^{63}$ M. Abbrescia ${ }^{64 a, 64 b}$ C. Calabria, ${ }^{64 a, 64 b}$ A. Colaleo, ${ }^{64 a}$ D. Creanza,${ }^{64 a, 64 c}$ L. Cristella, ${ }^{64 a, 64 b}$ N. De Filippis, ${ }^{64 a, 64 c}$ M. De Palma, ${ }^{64 a, 64 b}$ 
A. Di Florio, ${ }^{64 a, 64 b}$ F. Errico, ${ }^{64 a, 64 b}$ L. Fiore,${ }^{64 a}$ A. Gelmi ${ }^{64 a, 64 b}$ G. Iaselli, ${ }^{64 a, 64 c}$ M. Ince, ${ }^{64 a, 64 b}$ S. Lezki, ${ }^{64 a, 64 b}$ G. Maggi, ${ }^{64 a, 64 c}$ M. Maggi, ${ }^{64 a}$ G. Miniello, ${ }^{64 a, 64 b}$ S. My, ${ }^{64 a, 64 b}$ S. Nuzzo, ${ }^{64 a, 64 b}$ A. Pompili, ${ }^{64 a, 64 b}$ G. Pugliese,${ }^{64 a, 64 c}$ R. Radogna, ${ }^{64 a}$ A. Ranieri, ${ }^{64 a}$ G. Selvaggi, ${ }^{64 a, 64 b}$ A. Sharma,${ }^{64 a}$ L. Silvestris, ${ }^{64 a}$ R. Venditti, ${ }^{64 a}$ P. Verwilligen, ${ }^{64 a}$ G. Zito, ${ }^{64 a}$ G. Abbiendi, ${ }^{65 a}$ C. Battilana,${ }^{65 a, 65 b}$ D. Bonacorsi, ${ }^{65 a, 65 b}$ L. Borgonovi,${ }^{65 a, 65 b}$ S. Braibant-Giacomelli, ${ }^{65 a, 65 b}$ R. Campanini, ${ }^{65 a, 65 b}$ P. Capiluppi ${ }^{65 a, 65 b}$ A. Castro, ${ }^{65 a, 65 b}$ F. R. Cavallo, ${ }^{65 a}$ S. S. Chhibra,${ }^{65 a, 65 b}$ C. Ciocca ${ }^{65 a}$ G. Codispoti, ${ }^{65 a, 65 b}$ M. Cuffiani, ${ }^{65 a, 65 b}$ G. M. Dallavalle, ${ }^{65 a}$ F. Fabbri, ${ }^{65 a}$ A. Fanfani, ${ }^{65 a, 65 b}$ P. Giacomelli, ${ }^{65 a}$ C. Grandi, ${ }^{65 a}$ L. Guiducci, ${ }^{65 a, 65 b}$ F. Iemmi, ${ }^{65 a, 65 b}$ S. Marcellini, ${ }^{65 a}$ G. Masetti, ${ }^{65 a}$ A. Montanari, ${ }^{65 a}$ F. L. Navarria,${ }^{65 a, 65 b}$ A. Perrotta, ${ }^{65 a}$ F. Primavera ${ }^{65 a, 65 b, p}$ A. M. Rossi, ${ }^{65 a, 65 b}$ T. Rovelli, ${ }^{65 a, 65 b}$ G. P. Siroli, ${ }^{65,65 b}$ N. Tosi, ${ }^{65 a}$ S. Albergo, ${ }^{66 a, 66 b}$ A. Di Mattia, ${ }^{66 a}$ R. Potenza, ${ }^{66 a, 66 b}$ A. Tricomi, ${ }^{66 a, 66 b}$ C. Tuve, ${ }^{66 a, 66 b}$ G. Barbagli ${ }^{67 a}$ K. Chatterjee, ${ }^{67 a, 67 b}$ V. Ciulli, ${ }^{67 a, 67 b}$ C. Civinini,${ }^{67 a}$ R. D'Alessandro, ${ }^{67 a, 67 b}$ E. Focardi, ${ }^{67 a, 67 b}$ G. Latino, ${ }^{67 a}$ P. Lenzi, ${ }^{67 a, 67 b}$ M. Meschini, ${ }^{67 a}$ S. Paoletti, ${ }^{67 a}$ L. Russo, ${ }^{67 a, c c}$ G. Sguazzoni, ${ }^{67 a}$ D. Strom, ${ }^{67 a}$ L. Viliani, ${ }^{67 a}$ L. Benussi, ${ }^{68}$ S. Bianco, ${ }^{68}$ F. Fabbri, ${ }^{68}$ D. Piccolo, ${ }^{68}$ F. Ferro, ${ }^{69 \mathrm{a}}$ F. Ravera, ${ }^{69 \mathrm{a}, 69 \mathrm{~b}}$ E. Robutti, ${ }^{69 \mathrm{a}}$ S. Tosi,${ }^{69 \mathrm{a}, 69 \mathrm{~b}}$ A. Benaglia, ${ }^{70 \mathrm{a}}$ A. Beschi, ${ }^{70 a, 70 b}$ L. Brianza, ${ }^{70 a, 70 b}$ F. Brivio, ${ }^{70 a, 70 b}$ V. Ciriolo, ${ }^{70 a, 70 b, p}$ S. Di Guida, ${ }^{70 a, 70 b, p}$ M. E. Dinardo, ${ }^{70 a, 70 b}$

S. Fiorendi, ${ }^{70 a, 70 b}$ S. Gennai, ${ }^{70 a}$ A. Ghezzi, ${ }^{70 a, 70 b}$ P. Govoni ${ }^{70 a, 70 b}$ M. Malberti, ${ }^{70 a}, 70 b$ S. Malvezzi,${ }^{70 a}$ A. Massironi, ${ }^{70 a, 70 b}$ D. Menasce, ${ }^{70 a}$ L. Moroni, ${ }^{70 a}$ M. Paganoni, ${ }^{70 a, 70 b}$ D. Pedrini, ${ }^{70 a}$ S. Ragazzi, ${ }^{70 a, 70 b}$ T. Tabarelli de Fatis, ${ }^{70 a, 70 b}$ D. Zuolo, ${ }^{70 a}$ S. Buontempo, ${ }^{71 \mathrm{a}}$ N. Cavallo, ${ }^{71 \mathrm{a}, 71 \mathrm{c}}$ A. Di Crescenzo, ${ }^{71 a, 71 \mathrm{~b}}$ F. Fabozzi, ${ }^{71 \mathrm{a}, 71 \mathrm{c}}$ F. Fienga, ${ }^{71 \mathrm{a}}$ G. Galati, ${ }^{71 \mathrm{a}}$ A. O. M. Iorio, ${ }^{71 \mathrm{a}, 71 \mathrm{~b}}$ W. A. Khan, ${ }^{71 \mathrm{a}}$ L. Lista, ${ }^{71 \mathrm{a}}$ S. Meola, ${ }^{71 \mathrm{a}, 71 \mathrm{~d}, \mathrm{p}}$ P. Paolucci, ${ }^{71 \mathrm{a}, \mathrm{p}}$ C. Sciacca, ${ }^{71 \mathrm{a}, 7 \mathrm{~b}}$ E. Voevodina, ${ }^{71 \mathrm{a}, 71 \mathrm{~b}}$ P. Azzi ${ }^{72 \mathrm{a}}$ N. Bacchetta, ${ }^{72 a}$ A. Boletti, ${ }^{72 a, 72 b}$ A. Bragagnolo, ${ }^{72 a}$ R. Carlin, ${ }^{72 a, 72 b}$ P. Checchia, ${ }^{72 a}$ P. De Castro Manzano, ${ }^{72 a}$ T. Dorigo, ${ }^{72 a}$ U. Dosselli, ${ }^{72 a}$ F. Gasparini, ${ }^{72 a, 72 b}$ U. Gasparini, ${ }^{72 a, 72 b}$ A. Gozzelino, ${ }^{72 a}$ S. Y. Hoh, ${ }^{72 a}$ S. Lacaprara, ${ }^{72 a}$ P. Lujan, ${ }^{72 a}$ M. Margoni, ${ }^{72 a, 72 b}$ A. T. Meneguzzo, ${ }^{72 a, 72 b}$ J. Pazzini, ${ }^{72 a, 72 b}$ N. Pozzobon, ${ }^{72 a, 72 b}$ P. Ronchese, ${ }^{72 a, 72 b}$ R. Rossin, ${ }^{72 a, 72 b}$ F. Simonetto, ${ }^{72 a, 72 b}$ A. Tiko, ${ }^{72 a}$ E. Torassa, ${ }^{72 a}$ S. Ventura, ${ }^{72 a}$ M. Zanetti, ${ }^{72 a, 72 b}$ P. Zotto, ${ }^{72 a, 72 b}$ G. Zumerle, ${ }^{72 a, 72 b}$ A. Braghieri, ${ }^{73 a}$ A. Magnani, ${ }^{73 a}$ P. Montagna, ${ }^{73 a, 73 b}$ S. P. Ratti, ${ }^{73 a, 73 b}$ V. Re,${ }^{73 a}$ M. Ressegotti, ${ }^{73 a, 73 b}$ C. Riccardi, ${ }^{73 a, 73 b}$ P. Salvini, ${ }^{73 a}$ I. Vai, ${ }^{73 a, 73 b}$ P. Vitulo, ${ }^{73 a, 73 b}$ L. Alunni Solestizi, ${ }^{74 a, 74 b}$ M. Biasini,${ }^{74 a, 74 b}$ G. M. Bilei, ${ }^{74 a}$ C. Cecchi, ${ }^{74 a, 74 b}$ D. Ciangottini, ${ }^{74 a, 74 b}$ L. Fanò, ${ }^{74 a, 74 b}$ P. Lariccia, ${ }^{74 a, 74 b}$ R. Leonardi, ${ }^{74 a, 74 b}$ E. Manoni, ${ }^{74 a}$ G. Mantovani, ${ }^{74 a, 74 b}$ V. Mariani, ${ }^{74 a, 74 b}$ M. Menichelli, ${ }^{74 a}$ A. Rossi, ${ }^{74 a, 74 b}$ A. Santocchia,${ }^{74 a, 74 b}$ D. Spiga ${ }^{74 a}$ K. Androsov,${ }^{75 a}$ P. Azzurri, ${ }^{75 a}$ G. Bagliesi, ${ }^{75 a}$ L. Bianchini, ${ }^{75 a}$ T. Boccali, ${ }^{75 a}$ L. Borrello, ${ }^{75 a}$ R. Castaldi, ${ }^{75 a}$ M. A. Ciocci, ${ }^{75 a, 75 b}$ R. Dell'Orso, ${ }^{75 a}$ G. Fedi, ${ }^{75 a}$ F. Fiori, ${ }^{75 a, 75 c}$ L. Giannini, ${ }^{75 a, 75 c}$ A. Giassi, ${ }^{75 a}$ M. T. Grippo, ${ }^{75 a}$ F. Ligabue, ${ }^{75 a, 75 c}$ E. Manca, ${ }^{75 a, 75 c}$ G. Mandorli, ${ }^{75 a, 75 c}$ A. Messineo, ${ }^{75 a, 75 b}$ F. Palla, ${ }^{75 a}$ A. Rizzi, ${ }^{75 a, 75 b}$ P. Spagnolo, ${ }^{75 a}$ R. Tenchini, ${ }^{75 a}$ G. Tonelli, ${ }^{75 a, 75 b}$ A. Venturi, ${ }^{75 a}$ P. G. Verdini, ${ }^{75 a}$ L. Barone, ${ }^{76 a, 76 b}$

F. Cavallari, ${ }^{76 \mathrm{a}}$ M. Cipriani, ${ }^{76 a, 76 \mathrm{~b}}$ N. Daci ${ }^{76 \mathrm{a}}$ D. Del Re,${ }^{76 a, 76 b}$ E. Di Marco, ${ }^{76 a, 76 \mathrm{~b}}$ M. Diemoz, ${ }^{76 \mathrm{a}}$ S. Gelli, ${ }^{76 a, 76 \mathrm{~b}}$ E. Longo, ${ }^{76 a, 76 b}$ B. Marzocchi, ${ }^{76 a, 76 b}$ P. Meridiani, ${ }^{76 a}$ G. Organtini, ${ }^{76 a, 76 b}$ F. Pandolfi, ${ }^{76 a}$ R. Paramatti ${ }^{76 a, 76 b}$ F. Preiato, ${ }^{76 a, 76 b}$ S. Rahatlou, ${ }^{76 a, 76 b}$ C. Rovelli, ${ }^{76 a}$ F. Santanastasio, ${ }^{76 a, 76 b}$ N. Amapane, ${ }^{77 a, 77 b}$ R. Arcidiacono, ${ }^{77 a, 77 c}$ S. Argiro, ${ }^{77 a, 77 b}$

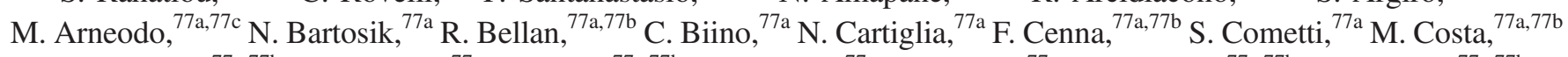
R. Covarelli, ${ }^{77,77 b}$ N. Demaria, ${ }^{77 a}$ B. Kiani, ${ }^{77 a, 77 b}$ C. Mariotti, ${ }^{77 a}$ S. Maselli, ${ }^{77 a}$ E. Migliore, ${ }^{77 a, 77 b}$ V. Monaco, ${ }^{77 a, 77 b}$

E. Monteil, ${ }^{77 a, 77 b}$ M. Monteno, ${ }^{77 a}$ M. M. Obertino, ${ }^{77 a, 77 b}$ L. Pacher, ${ }^{77 a, 77 b}$ N. Pastrone, ${ }^{77 a}$ M. Pelliccioni, ${ }^{77 a}$ G. L. Pinna Angioni, ${ }^{77 a, 77 b}$ A. Romero, ${ }^{77 a, 77 b}$ M. Ruspa, ${ }^{77 a, 77 c}$ R. Sacchi, ${ }^{77 a, 77 b}$ K. Shchelina, ${ }^{77 a, 77 b}$ V. Sola, ${ }^{77 a}$ A. Solano, ${ }^{77 a, 77 b}$ D. Soldi, ${ }^{77 a, 77 b}$ A. Staiano, ${ }^{77 a}$ S. Belforte, ${ }^{78 a}$ V. Candelise,${ }^{78 a, 78 b}$ M. Casarsa ${ }^{78 a}$ F. Cossutti, ${ }^{78 a}$ G. Della Ricca, ${ }^{78 a, 78 b}$ F. Vazzoler, ${ }^{78 a, 78 b}$ A. Zanetti, ${ }^{78 a}$ D. H. Kim, ${ }^{79}$ G. N. Kim, ${ }^{79}$ M. S. Kim, ${ }^{79}$ J. Lee, ${ }^{79}$ S. Lee, ${ }^{79}$ S. W. Lee, ${ }^{79}$ C. S. Moon, ${ }^{79}$ Y. D. Oh, ${ }^{79}$ S. Sekmen, ${ }^{79}$ D. C. Son, ${ }^{79}$ Y. C. Yang, ${ }^{79}$ H. Kim, ${ }^{80}$ D. H. Moon, ${ }^{80}$ G. Oh, ${ }^{80}$ J. Goh, ${ }^{81, d d}$ T. J. Kim, ${ }^{81}$ S. Cho, ${ }^{82}$ S. Choi, ${ }^{82}$ Y. Go, ${ }^{82}$ D. Gyun, ${ }^{82}$ S. Ha, ${ }^{82}$ B. Hong, ${ }^{82}$ Y. Jo, ${ }^{82}$ K. Lee,${ }^{82}$ K. S. Lee, ${ }^{82}$ S. Lee, ${ }^{82}$ J. Lim,${ }^{82}$ S. K. Park, ${ }^{82}$ Y. Roh, ${ }^{82}$ H. S. Kim, ${ }^{83}$ J. Almond, ${ }^{84}$ J. Kim, ${ }^{84}$ J. S. Kim, ${ }^{84}$ H. Lee, ${ }^{84}$ K. Lee,${ }^{84}$ K. Nam, ${ }^{84}$ S. B. Oh, ${ }^{84}$ B. C. Radburn-Smith, ${ }^{84}$ S. h. Seo ${ }^{84}$ U. K. Yang,${ }^{84}$ H. D. Yoo,${ }^{84}$ G. B. Yu, ${ }^{84}$ D. Jeon, ${ }^{85}$ H. Kim, ${ }^{85}$ J. H. Kim,${ }^{85}$ J. S. H. Lee,${ }^{85}$ I. C. Park,${ }^{85}$ Y. Choi, ${ }^{86}$ C. Hwang, ${ }^{86}$ J. Lee, ${ }^{86}$ I. Yu, ${ }^{86}$ V. Dudenas, ${ }^{87}$ A. Juodagalvis, ${ }^{87}$ J. Vaitkus, ${ }^{87}$ I. Ahmed, ${ }^{88}$ Z. A. Ibrahim ${ }^{88}$ M. A. B. Md Ali, ${ }^{88, \text { ee }}$ F. Mohamad Idris, ${ }^{88, f f}$ W. A. T. Wan Abdullah, ${ }^{88}$ M. N. Yusli, ${ }^{88}$ Z. Zolkapli, ${ }^{88}$

A. Castaneda Hernandez, ${ }^{89}$ J. A. Murillo Quijada,${ }^{89}$ H. Castilla-Valdez,${ }^{90}$ E. De La Cruz-Burelo, ${ }^{90}$ M. C. Duran-Osuna, ${ }^{90}$ I. Heredia-De La Cruz, ${ }^{90, g g}$ R. Lopez-Fernandez, ${ }^{90}$ J. Mejia Guisao, ${ }^{90}$ R. I. Rabadan-Trejo, ${ }^{90}$ M. Ramirez-Garcia, ${ }^{90}$ G. Ramirez-Sanchez, ${ }^{90}$ R. Reyes-Almanza, ${ }^{90}$ A. Sanchez-Hernandez,${ }^{90}$ S. Carrillo Moreno, ${ }^{91}$ C. Oropeza Barrera, ${ }^{91}$ F. Vazquez Valencia, ${ }^{91}$ J. Eysermans,${ }^{92}$ I. Pedraza,${ }^{92}$ H. A. Salazar Ibarguen, ${ }^{92}$ C. Uribe Estrada, ${ }^{92}$ A. Morelos Pineda, ${ }^{93}$ D. Krofcheck, ${ }^{94}$ S. Bheesette, ${ }^{95}$ P. H. Butler, ${ }^{95}$ A. Ahmad, ${ }^{96}$ M. Ahmad, ${ }^{96}$ M. I. Asghar, ${ }^{96}$ Q. Hassan, ${ }^{96}$ H. R. Hoorani,${ }^{96}$ S. Qazi ${ }^{96}$ M. A. Shah, ${ }^{96}$ M. Shoaib, ${ }^{96}$ M. Waqas, ${ }^{96}$ H. Bialkowska,,${ }^{97}$ M. Bluj,,${ }^{97}$ B. Boimska, ${ }^{97}$ T. Frueboes, ${ }^{97}$ M. Górski, ${ }^{97}$ M. Kazana, ${ }^{97}$ K. Nawrocki, ${ }^{97}$ M. Szleper, ${ }^{97}$ P. Traczyk, ${ }^{97}$ P. Zalewski, ${ }^{97}$ K. Bunkowski, ${ }^{98}$ A. Byszuk, ${ }^{98, h h}$ K. Doroba, ${ }^{98}$ 
A. Kalinowski, ${ }^{98}$ M. Konecki, ${ }^{98}$ J. Krolikowski, ${ }^{98}$ M. Misiura, ${ }^{98}$ M. Olszewski, ${ }^{98}$ A. Pyskir, ${ }^{98}$ M. Walczak, ${ }^{98}$ M. Araujo, ${ }^{99}$ P. Bargassa, ${ }^{99}$ C. Beirão Da Cruz E Silva, ${ }^{99}$ A. Di Francesco,${ }^{99}$ P. Faccioli,${ }^{99}$ B. Galinhas, ${ }^{99}$ M. Gallinaro, ${ }^{99}$ J. Hollar, ${ }^{99}$ N. Leonardo, ${ }^{99}$ M. V. Nemallapudi, ${ }^{99}$ J. Seixas, ${ }^{99}$ G. Strong, ${ }^{99}$ O. Toldaiev, ${ }^{99}$ D. Vadruccio, ${ }^{99}$ J. Varela, ${ }^{99}$ S. Afanasiev, ${ }^{100}$ V. Alexakhin, ${ }^{100}$ P. Bunin, ${ }^{100}$ M. Gavrilenko, ${ }^{100}$ A. Golunov, ${ }^{100}$ I. Golutvin, ${ }^{100}$ N. Gorbounov, ${ }^{100}$ V. Karjavin, ${ }^{100}$ A. Lanev, ${ }^{100}$ A. Malakhov, ${ }^{100}$ V. Matveev, ${ }^{100, i i, j j}$ P. Moisenz, ${ }^{100}$ V. Palichik, ${ }^{100}$ V. Perelygin,,${ }^{100}$ M. Savina, ${ }^{100}$ S. Shmatov, ${ }^{100}$ V. Smirnov, ${ }^{100}$ N. Voytishin, ${ }^{100}$ A. Zarubin, ${ }^{100}$ V. Golovtsov,${ }^{101}$ Y. Ivanov, ${ }^{101}$ V. Kim,${ }^{101, k k}$ E. Kuznetsova, ${ }^{101,11}$ P. Levchenko, ${ }^{101}$ V. Murzin, ${ }^{101}$ V. Oreshkin, ${ }^{101}$ I. Smirnov, ${ }^{101}$ D. Sosnov, ${ }^{101}$ V. Sulimov, ${ }^{101}$ L. Uvarov, ${ }^{101}$ S. Vavilov, ${ }^{101}$ A. Vorobyev, ${ }^{101}$ Yu. Andreev, ${ }^{102}$ A. Dermenev, ${ }^{102}$ S. Gninenko, ${ }^{102}$ N. Golubev, ${ }^{102}$ A. Karneyeu, ${ }^{102}$ M. Kirsanov, ${ }^{102}$ N. Krasnikov, ${ }^{102}$ A. Pashenkov, ${ }^{102}$ D. Tlisov, ${ }^{102}$ A. Toropin, ${ }^{102}$ V. Epshteyn, ${ }^{103}$ V. Gavrilov, ${ }^{103}$ N. Lychkovskaya, ${ }^{103}$ V. Popov, ${ }^{103}$ I. Pozdnyakov, ${ }^{103}$ G. Safronov, ${ }^{103}$ A. Spiridonov, ${ }^{103}$ A. Stepennov, ${ }^{103}$ V. Stolin, ${ }^{103}$ M. Toms,${ }^{103}$ E. Vlasov, ${ }^{103}$ A. Zhokin, ${ }^{103}$ T. Aushev, ${ }^{104}$ R. Chistov, ${ }^{105, \mathrm{~mm}}$ M. Danilov, ${ }^{105, \mathrm{~mm}}$ P. Parygin, ${ }^{105}$ D. Philippov, ${ }^{105} \mathrm{~S}$. Polikarpov, ${ }^{105, \mathrm{~mm}}$ E. Tarkovskii, ${ }^{105}$ V. Andreev, ${ }^{106}$ M. Azarkin, ${ }^{106, j \mathrm{j}}$ I. Dremin,,${ }^{106, j \mathrm{jj}}$ M. Kirakosyan, ${ }^{106, j \mathrm{jj}}$ S. V. Rusakov, ${ }^{106}$ A. Terkulov, ${ }^{106}$ A. Baskakov, ${ }^{107}$ A. Belyaev, ${ }^{107}$ E. Boos, ${ }^{107}$ M. Dubinin, ${ }^{107, n n}$ L. Dudko, ${ }^{107}$ A. Ershov, ${ }^{107}$ A. Gribushin, ${ }^{107}$ V. Klyukhin, ${ }^{107}$ O. Kodolova, ${ }^{107}$ I. Lokhtin, ${ }^{107}$ I. Miagkov, ${ }^{107}$ S. Obraztsov, ${ }^{107}$ S. Petrushanko, ${ }^{107}$ V. Savrin, ${ }^{107}$ A. Snigirev, ${ }^{107}$ V. Blinov, ${ }^{108,00}$ T. Dimova, ${ }^{108,00}$ L. Kardapoltsev, ${ }^{108,00}$ D. Shtol, ${ }^{108,00}$ Y. Skovpen, ${ }^{108,00}$ I. Azhgirey, ${ }^{109}$ I. Bayshev,${ }^{109}$ S. Bitioukov, ${ }^{109}$ D. Elumakhov, ${ }^{109}$ A. Godizov, ${ }^{109}$ V. Kachanov, ${ }^{109}$ A. Kalinin, ${ }^{109}$ D. Konstantinov, ${ }^{109}$ P. Mandrik, ${ }^{109}$ V. Petrov, ${ }^{109}$ R. Ryutin, ${ }^{109}$ S. Slabospitskii, ${ }^{109}$ A. Sobol, ${ }^{109}$ S. Troshin, ${ }^{109}$ N. Tyurin, ${ }^{109}$ A. Uzunian, ${ }^{109}$ A. Volkov, ${ }^{109}$ A. Babaev, ${ }^{110}$ S. Baidali, ${ }^{110}$ V. Okhotnikov, ${ }^{110}$ P. Adzic, ${ }^{11, p p}$ P. Cirkovic, ${ }^{111}$ D. Devetak, ${ }^{111}$ M. Dordevic, ${ }^{111}$ J. Milosevic, ${ }^{111}$ J. Alcaraz Maestre, ${ }^{112}$ A. Álvarez Fernández, ${ }^{112}$ I. Bachiller, ${ }^{112}$ M. Barrio Luna, ${ }^{112}$ J. A. Brochero Cifuentes, ${ }^{112}$ M. Cerrada, ${ }^{112}$ N. Colino, ${ }^{112}$ B. De La Cruz, ${ }^{12}$ A. Delgado Peris, ${ }^{112}$ C. Fernandez Bedoya, ${ }^{112}$ J. P. Fernández Ramos, ${ }^{112}$ J. Flix, ${ }^{112}$ M. C. Fouz, ${ }^{112}$ O. Gonzalez Lopez, ${ }^{112}$ S. Goy Lopez, ${ }^{112}$ J. M. Hernandez, ${ }^{112}$ M. I. Josa, ${ }^{112}$ D. Moran, ${ }^{112}$ A. Pérez-Calero Yzquierdo, ${ }^{112}$ J. Puerta Pelayo, ${ }^{112}$ I. Redondo, ${ }^{112}$ L. Romero, ${ }^{112}$ M. S. Soares, ${ }^{112}$ A. Triossi, ${ }^{112}$ C. Albajar, ${ }^{113}$ J. F. de Trocóniz, ${ }^{113}$ J. Cuevas, ${ }^{114}$ C. Erice, ${ }^{114}$ J. Fernandez Menendez, ${ }^{114}$ S. Folgueras, ${ }^{114}$ I. Gonzalez Caballero, ${ }^{114}$ J. R. González Fernández,${ }^{114}$ E. Palencia Cortezon, ${ }^{114}$ V. Rodríguez Bouza, ${ }^{114}$ S. Sanchez Cruz, ${ }^{114}$ P. Vischia, ${ }^{114}$ J. M. Vizan Garcia, ${ }^{114}$ I. J. Cabrillo, ${ }^{115}$ A. Calderon, ${ }^{115}$ B. Chazin Quero, ${ }^{115}$ J. Duarte Campderros, ${ }^{115}$ M. Fernandez, ${ }^{115}$ P. J. Fernández Manteca, ${ }^{115}$ A. García Alonso, ${ }^{115}$ J. Garcia-Ferrero, ${ }^{115}$ G. Gomez, ${ }^{115}$ A. Lopez Virto, ${ }^{115}$ J. Marco, ${ }^{115}$ C. Martinez Rivero, ${ }^{115}$ P. Martinez Ruiz del Arbol, ${ }^{115}$ F. Matorras, ${ }^{115}$ J. Piedra Gomez, ${ }^{115}$ C. Prieels, ${ }^{115}$ T. Rodrigo, ${ }^{115}$ A. Ruiz-Jimeno, ${ }^{115}$ L. Scodellaro, ${ }^{115}$ N. Trevisani, ${ }^{115}$ I. Vila, ${ }^{115}$ R. Vilar Cortabitarte, ${ }^{115}$ D. Abbaneo, ${ }^{116}$ B. Akgun, ${ }^{116}$ E. Auffray, ${ }^{116}$ P. Baillon, ${ }^{116}$ A. H. Ball, ${ }^{116}$ D. Barney, ${ }^{116}$ J. Bendavid, ${ }^{116}$ M. Bianco, ${ }^{116}$ A. Bocci, ${ }^{116}$ C. Botta, ${ }^{116}$ E. Brondolin, ${ }^{116}$ T. Camporesi, ${ }^{116}$ M. Cepeda, ${ }^{116}$ G. Cerminara,${ }^{116}$ E. Chapon, ${ }^{116}$ Y. Chen, ${ }^{116}$ G. Cucciati, ${ }^{116}$ D. d'Enterria, ${ }^{116}$ A. Dabrowski, ${ }^{116}$ V. Daponte, ${ }^{116}$ A. David, ${ }^{116}$ A. De Roeck, ${ }^{116}$ N. Deelen, ${ }^{116}$ M. Dobson, ${ }^{116}$ M. Dünser,${ }^{116}$ N. Dupont, ${ }^{116}$ A. Elliott-Peisert, ${ }^{116}$ P. Everaerts, ${ }^{116}$ F. Fallavollita, ${ }^{16, q q}$ D. Fasanella, ${ }^{116}$ G. Franzoni, ${ }^{116}$ J. Fulcher, ${ }^{116}$ W. Funk, ${ }^{116}$ D. Gigi, ${ }^{116}$ A. Gilbert, ${ }^{116}$ K. Gill, ${ }^{116}$ F. Glege,${ }^{116}$ M. Guilbaud, ${ }^{116}$ D. Gulhan, ${ }^{116}$ J. Hegeman, ${ }^{116}$ V. Innocente,${ }^{116}$ A. Jafari, ${ }^{116}$ P. Janot, ${ }^{116}$ O. Karacheban, ${ }^{116, \mathrm{~s}}$ J. Kieseler, ${ }^{116}$ A. Kornmayer, ${ }^{116}$ M. Krammer, ${ }^{116, b}$ C. Lange, ${ }^{116}$ P. Lecoq, ${ }^{116}$ C. Lourenço, ${ }^{116}$ L. Malgeri, ${ }^{116}$ M. Mannelli, ${ }^{116}$ F. Meijers, ${ }^{116}$ J. A. Merlin, ${ }^{116}$ S. Mersi, ${ }^{116}$ E. Meschi, ${ }^{116}$ P. Milenovic, ${ }^{116, r r}$ F. Moortgat, ${ }^{116}$ M. Mulders, ${ }^{116}$ J. Ngadiuba, ${ }^{116}$ S. Nourbakhsh,${ }^{116}$ S. Orfanelli, ${ }^{116}$ L. Orsini, ${ }^{116}$ F. Pantaleo, ${ }^{116, p}$ L. Pape, ${ }^{116}$ E. Perez, ${ }^{116}$ M. Peruzzi, ${ }^{116}$ A. Petrilli, ${ }^{116}$ G. Petrucciani, ${ }^{116}$ A. Pfeiffer, ${ }^{116}$ M. Pierini, ${ }^{116}$ F. M. Pitters, ${ }^{116}$ D. Rabady, ${ }^{116}$ A. Racz, ${ }^{116}$ T. Reis, ${ }^{116}$ G. Rolandi, ${ }^{116, s s}$ M. Rovere, ${ }^{116}$ H. Sakulin, ${ }^{116}$ C. Schäfer,${ }^{116}$ C. Schwick, ${ }^{116}$ M. Seidel, ${ }^{116}$ M. Selvaggi, ${ }^{116}$ A. Sharma, ${ }^{116}$ P. Silva, ${ }^{116}$ P. Sphicas, ${ }^{116, t t}$ A. Stakia, ${ }^{116}$ J. Steggemann, ${ }^{116}$ M. Tosi, ${ }^{116}$ D. Treille, ${ }^{116}$ A. Tsirou, ${ }^{116}$ V. Veckalns, ${ }^{116, \text { uu }}$ W. D. Zeuner, ${ }^{116}$ L. Caminada, ${ }^{117, v v}$ K. Deiters,${ }^{117}$ W. Erdmann, ${ }^{117}$ R. Horisberger, ${ }^{117}$ Q. Ingram, ${ }^{117}$ H. C. Kaestli, ${ }^{117}$ D. Kotlinski, ${ }^{117}$ U. Langenegger, ${ }^{117}$ T. Rohe, ${ }^{117}$ S. A. Wiederkehr, ${ }^{117}$ M. Backhaus, ${ }^{118}$ L. Bäni, ${ }^{118}$ P. Berger, ${ }^{118}$ N. Chernyavskaya, ${ }^{118}$ G. Dissertori, ${ }^{118}$ M. Dittmar, ${ }^{118}$ M. Donegà, ${ }^{118}$ C. Dorfer, ${ }^{118}$ C. Grab,${ }^{118}$ C. Heidegger, ${ }^{118}$ D. Hits, ${ }^{118}$ J. Hoss, ${ }^{118}$ T. Klijnsma, ${ }^{118}$ W. Lustermann, ${ }^{118}$ R. A. Manzoni, ${ }^{118}$ M. Marionneau, ${ }^{118}$ M. T. Meinhard, ${ }^{118}$ F. Micheli, ${ }^{118}$ P. Musella, ${ }^{118}$ F. Nessi-Tedaldi, ${ }^{118}$ J. Pata, ${ }^{118}$ F. Pauss, ${ }^{118}$ G. Perrin, ${ }^{118}$ L. Perrozzi, ${ }^{118}$ S. Pigazzini, ${ }^{118}$ M. Quittnat, ${ }^{118}$ D. Ruini, ${ }^{118}$ D. A. Sanz Becerra, ${ }^{118}$ M. Schönenberger, ${ }^{118}$ L. Shchutska, ${ }^{118}$ V. R. Tavolaro, ${ }^{118}$

K. Theofilatos, ${ }^{118}$ M. L. Vesterbacka Olsson, ${ }^{118}$ R. Wallny, ${ }^{118}$ D. H. Zhu, ${ }^{118}$ T. K. Aarrestad,${ }^{119}$ C. Amsler, ${ }^{19, \text { ww }}$ D. Brzhechko, ${ }^{119}$ M. F. Canelli, ${ }^{119}$ A. De Cosa,${ }^{119}$ R. Del Burgo, ${ }^{119}$ S. Donato, ${ }^{119}$ C. Galloni, ${ }^{119}$ T. Hreus, ${ }^{119}$ B. Kilminster, ${ }^{119}$ I. Neutelings, ${ }^{119}$ D. Pinna, ${ }^{119}$ G. Rauco, ${ }^{119}$ P. Robmann, ${ }^{119}$ D. Salerno, ${ }^{119}$ K. Schweiger,${ }^{119}$ C. Seitz, ${ }^{119}$ Y. Takahashi, ${ }^{119}$ A. Zucchetta, ${ }^{119}$ Y. H. Chang, ${ }^{120}$ K. y. Cheng, ${ }^{120}$ T. H. Doan, ${ }^{120}$ Sh. Jain, ${ }^{120}$ R. Khurana, ${ }^{120}$ C. M. Kuo, ${ }^{120}$ 
W. Lin, ${ }^{120}$ A. Pozdnyakov, ${ }^{120}$ S. S. Yu, ${ }^{120}$ P. Chang, ${ }^{121}$ Y. Chao, ${ }^{121}$ K. F. Chen, ${ }^{121}$ P. H. Chen, ${ }^{121}$ W.-S. Hou, ${ }^{121}$ Arun Kumar, ${ }^{121}$ Y. y. Li, ${ }^{121}$ Y. F. Liu, ${ }^{121}$ R.-S. Lu, ${ }^{121}$ E. Paganis, ${ }^{121}$ A. Psallidas, ${ }^{121}$ A. Steen, ${ }^{121}$ B. Asavapibhop, ${ }^{122}$ N. Srimanobhas, ${ }^{122}$ N. Suwonjandee, ${ }^{122}$ A. Bat, ${ }^{123}$ F. Boran, ${ }^{123}$ S. Cerci, ${ }^{123, x x}$ S. Damarseckin, ${ }^{123}$ Z. S. Demiroglu, ${ }^{123}$ F. Dolek, ${ }^{123}$ C. Dozen, ${ }^{123}$ I. Dumanoglu, ${ }^{123}$ S. Girgis, ${ }^{123}$ G. Gokbulut, ${ }^{123}$ Y. Guler, ${ }^{123}$ E. Gurpinar, ${ }^{123}$ I. Hos, ${ }^{123, y y}$ C. Isik, ${ }^{123}$ E. E. Kangal, ${ }^{123, z z}$ O. Kara, ${ }^{123}$ A. Kayis Topaksu, ${ }^{123}$ U. Kiminsu, ${ }^{123}$ M. Oglakci, ${ }^{123}$ G. Onengut, ${ }^{123}$ K. Ozdemir, ${ }^{123, \text { aaa }}$ S. Ozturk, ${ }^{123, \text { bbb }}$ A. Polatoz, ${ }^{123}$ B. Tali, ${ }^{123, x x}$ U. G. Tok, ${ }^{123}$ S. Turkcapar, ${ }^{123}$ I. S. Zorbakir, ${ }^{123}$ C. Zorbilmez, ${ }^{123}$ B. Isildak, ${ }^{124, c c c}$ G. Karapinar, ${ }^{124, \text { ddd }}$ M. Yalvac, ${ }^{124}$ M. Zeyrek, ${ }^{124}$ I. O. Atakisi, ${ }^{125}$ E. Gülmez, ${ }^{125}$ M. Kaya, ${ }^{125, \text { eee }}$ O. Kaya, ${ }^{125, \text { fff }}$ S. Ozkorucuklu, ${ }^{125, \text { ggg }}$ S. Tekten, ${ }^{125}$ E. A. Yetkin, ${ }^{125, \text { hhh }}$ M. N. Agaras, ${ }^{126}$ S. Atay, ${ }^{126}$ A. Cakir, ${ }^{126}$ K. Cankocak, ${ }^{126}$ Y. Komurcu, ${ }^{126}$ S. Sen, ${ }^{126, i i i}$ B. Grynyov, ${ }^{127}$ L. Levchuk, ${ }^{128}$ F. Ball, ${ }^{129}$ L. Beck, ${ }^{129}$ J. J. Brooke, ${ }^{129}$ D. Burns,${ }^{129}$ E. Clement, ${ }^{129}$ D. Cussans, ${ }^{129}$ O. Davignon, ${ }^{129}$ H. Flacher, ${ }^{129}$ J. Goldstein, ${ }^{129}$ G. P. Heath, ${ }^{129}$ H. F. Heath, ${ }^{129}$ L. Kreczko, ${ }^{129}$ D. M. Newbold ${ }^{129, j \mathrm{jj}}$ S. Paramesvaran, ${ }^{129}$ B. Penning, ${ }^{129}$ T. Sakuma, ${ }^{129}$ D. Smith, ${ }^{129}$ V. J. Smith, ${ }^{129}$ J. Taylor, ${ }^{129}$ A. Titterton, ${ }^{129}$ K. W. Bell, ${ }^{130}$ A. Belyaev, ${ }^{130, k k k}$ C. Brew, ${ }^{130}$ R. M. Brown, ${ }^{130}$ D. Cieri, ${ }^{130}$ D. J. A. Cockerill, ${ }^{130}$ J. A. Coughlan, ${ }^{130}$ K. Harder, ${ }^{130}$ S. Harper, ${ }^{130}$ J. Linacre, ${ }^{130}$ E. Olaiya, ${ }^{130}$ D. Petyt, ${ }^{130}$ C. H. Shepherd-Themistocleous, ${ }^{130}$ A. Thea, ${ }^{130}$ I. R. Tomalin, ${ }^{130}$ T. Williams, ${ }^{130}$ W. J. Womersley ${ }^{130}$ G. Auzinger, ${ }^{131}$ R. Bainbridge, ${ }^{131}$ P. Bloch, ${ }^{131}$ J. Borg, ${ }^{131}$ S. Breeze, ${ }^{131}$ O. Buchmuller, ${ }^{131}$ A. Bundock, ${ }^{131}$ S. Casasso, ${ }^{131}$ D. Colling, ${ }^{131}$ L. Corpe,${ }^{131}$ P. Dauncey,${ }^{131}$ G. Davies,${ }^{131}$ M. Della Negra, ${ }^{131}$ R. Di Maria ${ }^{131}$ Y. Haddad, ${ }^{131}$ G. Hall, ${ }^{131}$ G. Iles, ${ }^{131}$ T. James, ${ }^{131}$ M. Komm, ${ }^{131}$ C. Laner, ${ }^{131}$ L. Lyons, ${ }^{131}$ A.-M. Magnan, ${ }^{131}$ S. Malik, ${ }^{131}$ A. Martelli, ${ }^{131}$ J. Nash, ${ }^{131,111}$ A. Nikitenko, ${ }^{131, h}$ V. Palladino, ${ }^{131}$ M. Pesaresi, ${ }^{131}$ A. Richards, ${ }^{131}$ A. Rose, ${ }^{131}$ E. Scott, ${ }^{131}$ C. Seez, ${ }^{131}$ A. Shtipliyski, ${ }^{131}$ G. Singh, ${ }^{131}$ M. Stoye, ${ }^{131}$ T. Strebler, ${ }^{131}$ S. Summers, ${ }^{131}$ A. Tapper, ${ }^{131}$ K. Uchida, ${ }^{131}$ T. Virdee, ${ }^{131, p}$ N. Wardle, ${ }^{131}$ D. Winterbottom, ${ }^{131}$ J. Wright, ${ }^{131}$ S. C. Zenz, ${ }^{131}$ J. E. Cole, ${ }^{132}$ P. R. Hobson, ${ }^{132}$ A. Khan, ${ }^{132}$ P. Kyberd, ${ }^{132}$ C. K. Mackay, ${ }^{132}$ A. Morton ${ }^{132}$ I. D. Reid, ${ }^{132}$ L. Teodorescu, ${ }^{132}$ S. Zahid, ${ }^{132}$ K. Call, ${ }^{133}$ J. Dittmann, ${ }^{133}$ K. Hatakeyama, ${ }^{133}$ H. Liu, ${ }^{133}$ C. Madrid, ${ }^{133}$ B. Mcmaster, ${ }^{133}$ N. Pastika, ${ }^{133}$ C. Smith, ${ }^{133}$ R. Bartek ${ }^{134}$ A. Dominguez, ${ }^{134}$ A. Buccilli, ${ }^{135}$ S. I. Cooper, ${ }^{135}$ C. Henderson, ${ }^{135}$ P. Rumerio, ${ }^{135}$ C. West,${ }^{135}$ D. Arcaro, ${ }^{136}$ T. Bose, ${ }^{136}$ D. Gastler, ${ }^{136}$ D. Rankin, ${ }^{136}$ C. Richardson, ${ }^{136}$ J. Rohlf, ${ }^{136}$ L. Sulak, ${ }^{136}$ D. Zou, ${ }^{136}$ G. Benelli, ${ }^{137}$ X. Coubez, ${ }^{137}$ D. Cutts, ${ }^{137}$ M. Hadley, ${ }^{137}$ J. Hakala, ${ }^{137}$ U. Heintz, ${ }^{137}$ J. M. Hogan, ${ }^{137, \mathrm{mmm}}$ K. H. M. Kwok, ${ }^{137}$ E. Laird, ${ }^{137}$ G. Landsberg, ${ }^{137}$ J. Lee, ${ }^{137}$ Z. Mao ${ }^{137}$ M. Narain, ${ }^{137}$ S. Piperov, ${ }^{137}$ S. Sagir, ${ }^{137, n n n}$ R. Syarif, ${ }^{137}$ E. Usai, ${ }^{137}$ D. Yu, ${ }^{137}$ R. Band, ${ }^{138}$ C. Brainerd, ${ }^{138}$ R. Breedon, ${ }^{138}$ D. Burns, ${ }^{138}$ M. Calderon De La Barca Sanchez, ${ }^{138}$ M. Chertok, ${ }^{138}$ J. Conway, ${ }^{138}$ R. Conway, ${ }^{138}$ P. T. Cox, ${ }^{138}$ R. Erbacher, ${ }^{138}$ C. Flores, ${ }^{138}$ G. Funk, ${ }^{138}$ W. Ko, ${ }^{138}$ O. Kukral, ${ }^{138}$ R. Lander,${ }^{138}$ M. Mulhearn, ${ }^{138}$ D. Pellett, ${ }^{138}$ J. Pilot, ${ }^{138}$ S. Shalhout, ${ }^{138}$ M. Shi, ${ }^{138}$ D. Stolp ${ }^{138}$ D. Taylor, ${ }^{138}$ K. Tos, ${ }^{138}$ M. Tripathi, ${ }^{138}$ Z. Wang ${ }^{138}$ F. Zhang, ${ }^{138}$ M. Bachtis, ${ }^{139}$ C. Bravo, ${ }^{139}$ R. Cousins, ${ }^{139}$ A. Dasgupta, ${ }^{139}$ A. Florent,${ }^{139}$ J. Hauser, ${ }^{139}$ M. Ignatenko, ${ }^{139}$ N. Mccoll, ${ }^{139}$ S. Regnard, ${ }^{139}$ D. Saltzberg, ${ }^{139}$ C. Schnaible, ${ }^{139}$ V. Valuev, ${ }^{139}$ E. Bouvier, ${ }^{140}$ K. Burt, ${ }^{140}$ R. Clare, ${ }^{140}$ J. W. Gary, ${ }^{140}$

S. M. A. Ghiasi Shirazi, ${ }^{140}$ G. Hanson, ${ }^{140}$ G. Karapostoli, ${ }^{140}$ E. Kennedy, ${ }^{140}$ F. Lacroix, ${ }^{140}$ O. R. Long, ${ }^{140}$

M. Olmedo Negrete, ${ }^{140}$ M. I. Paneva, ${ }^{140}$ W. Si,${ }^{140}$ L. Wang, ${ }^{140}$ H. Wei, ${ }^{140}$ S. Wimpenny, ${ }^{140}$ B. R. Yates, ${ }^{140}$ J. G. Branson, ${ }^{141}$ S. Cittolin, ${ }^{141}$ M. Derdzinski, ${ }^{141}$ R. Gerosa,${ }^{141}$ D. Gilbert, ${ }^{141}$ B. Hashemi,${ }^{141}$ A. Holzner,${ }^{141}$ D. Klein, ${ }^{141}$ G. Kole, ${ }^{141}$ V. Krutelyov, ${ }^{141}$ J. Letts, ${ }^{141}$ M. Masciovecchio, ${ }^{141}$ D. Olivito, ${ }^{141}$ S. Padhi, ${ }^{141}$ M. Pieri, ${ }^{141}$ M. Sani, ${ }^{141}$ V. Sharma, ${ }^{141}$ S. Simon, ${ }^{141}$ M. Tadel, ${ }^{141}$ A. Vartak, ${ }^{141}$ S. Wasserbaech, ${ }^{141,000}$ J. Wood,${ }^{141}$ F. Würthwein, ${ }^{141}$ A. Yagil, ${ }^{141}$ G. Zevi Della Porta, ${ }^{141}$ N. Amin, ${ }^{142}$ R. Bhandari, ${ }^{142}$ J. Bradmiller-Feld, ${ }^{142}$ C. Campagnari, ${ }^{142}$ M. Citron, ${ }^{142}$ A. Dishaw, ${ }^{142}$ V. Dutta, ${ }^{142}$ M. Franco Sevilla, ${ }^{142}$ L. Gouskos, ${ }^{142}$ R. Heller, ${ }^{142}$ J. Incandela, ${ }^{142}$ A. Ovcharova, ${ }^{142}$ H. Qu, ${ }^{142}$ J. Richman, ${ }^{142}$ D. Stuart, ${ }^{142}$ I. Suarez, ${ }^{142}$ S. Wang, ${ }^{142}$ J. Yoo, ${ }^{142}$ D. Anderson, ${ }^{143}$ A. Bornheim, ${ }^{143}$ J. M. Lawhorn, ${ }^{143}$ H. B. Newman, ${ }^{143}$ T. Q. Nguyen, ${ }^{143}$ M. Spiropulu, ${ }^{143}$ J. R. Vlimant, ${ }^{143}$ R. Wilkinson, ${ }^{143}$ S. Xie, ${ }^{143}$ Z. Zhang, ${ }^{143}$ R. Y. Zhu, ${ }^{143}$ M. B. Andrews, ${ }^{144}$ T. Ferguson, ${ }^{144}$ T. Mudholkar, ${ }^{144}$ M. Paulini, ${ }^{144}$ M. Sun, ${ }^{144}$ I. Vorobiev, ${ }^{144}$ M. Weinberg, ${ }^{144}$ J. P. Cumalat,${ }^{145}$ W. T. Ford, ${ }^{145}$ F. Jensen, ${ }^{145}$ A. Johnson, ${ }^{145}$ M. Krohn, ${ }^{145}$ S. Leontsinis, ${ }^{145}$ E. MacDonald, ${ }^{145}$ T. Mulholland, ${ }^{145}$ K. Stenson, ${ }^{145}$ K. A. Ulmer, ${ }^{145}$ S. R. Wagner, ${ }^{145}$ J. Alexander,${ }^{146}$ J. Chaves, ${ }^{146}$ Y. Cheng, ${ }^{146}$ J. Chu, ${ }^{146}$ A. Datta, ${ }^{146}$ K. Mcdermott, ${ }^{146}$ N. Mirman, ${ }^{146}$ J. R. Patterson, ${ }^{146}$ D. Quach, ${ }^{146}$ A. Rinkevicius, ${ }^{146}$ A. Ryd, ${ }^{146}$ L. Skinnari, ${ }^{146}$ L. Soffi, ${ }^{146}$ S. M. Tan, ${ }^{146}$ Z. Tao, ${ }^{146}$ J. Thom,${ }^{146}$ J. Tucker, ${ }^{146}$ P. Wittich,${ }^{146}$ M. Zientek, ${ }^{146}$ S. Abdullin, ${ }^{147}$ M. Albrow, ${ }^{147}$ M. Alyari, ${ }^{147}$ G. Apollinari, ${ }^{147}$ A. Apresyan, ${ }^{147}$ A. Apyan,${ }^{147}$ S. Banerjee,${ }^{147}$ L. A. T. Bauerdick, ${ }^{147}$ A. Beretvas,${ }^{147}$ J. Berryhill, ${ }^{147}$ P. C. Bhat, ${ }^{147}$ G. Bolla, ${ }^{147, a}$ K. Burkett, ${ }^{147}$ J. N. Butler, ${ }^{147}$ A. Canepa,${ }^{147}$ G. B. Cerati, ${ }^{147}$ H. W. K. Cheung, ${ }^{147}$ F. Chlebana, ${ }^{147}$ M. Cremonesi ${ }^{147}$ J. Duarte, ${ }^{147}$ V. D. Elvira ${ }^{147}$ J. Freeman, ${ }^{147}$ Z. Gecse, ${ }^{147}$ E. Gottschalk, ${ }^{147}$ L. Gray, ${ }^{147}$ D. Green, ${ }^{147}$ S. Grünendahl, ${ }^{147}$ O. Gutsche, ${ }^{147}$ J. Hanlon, ${ }^{147}$ R. M. Harris, ${ }^{147}$ S. Hasegawa, ${ }^{147}$ J. Hirschauer, ${ }^{147}$ Z. Hu, ${ }^{147}$ B. Jayatilaka, ${ }^{147}$ 
S. Jindariani, ${ }^{147}$ M. Johnson, ${ }^{147}$ U. Joshi, ${ }^{147}$ B. Klima, ${ }^{147}$ M. J. Kortelainen, ${ }^{147}$ B. Kreis, ${ }^{147}$ S. Lammel,${ }^{147}$ D. Lincoln, ${ }^{147}$ R. Lipton, ${ }^{147}$ M. Liu, ${ }^{147}$ T. Liu, ${ }^{147}$ J. Lykken, ${ }^{147}$ K. Maeshima, ${ }^{147}$ J. M. Marraffino, ${ }^{147}$ D. Mason, ${ }^{147}$ P. McBride, ${ }^{147}$ P. Merkel, ${ }^{147}$ S. Mrenna, ${ }^{147}$ S. Nahn, ${ }^{147}$ V. O'Dell ${ }^{147}$ K. Pedro, ${ }^{147}$ C. Pena,${ }^{147}$ O. Prokofyev, ${ }^{147}$ G. Rakness,${ }^{147}$ L. Ristori, ${ }^{147}$ A. Savoy-Navarro, ${ }^{147, \text { ppp }}$ B. Schneider, ${ }^{147}$ E. Sexton-Kennedy, ${ }^{147}$ A. Soha, ${ }^{147}$ W. J. Spalding, ${ }^{147}$ L. Spiegel, ${ }^{147}$ S. Stoynev, ${ }^{147}$ J. Strait, ${ }^{147}$ N. Strobbe, ${ }^{147}$ L. Taylor, ${ }^{147}$ S. Tkaczyk, ${ }^{147}$ N. V. Tran, ${ }^{147}$ L. Uplegger, ${ }^{147}$ E. W. Vaandering, ${ }^{147}$ C. Vernieri, ${ }^{147}$ M. Verzocchi, ${ }^{147}$ R. Vidal, ${ }^{147}$ M. Wang,${ }^{147}$ H. A. Weber ${ }^{147}$ A. Whitbeck,${ }^{147}$ D. Acosta, ${ }^{148}$ P. Avery, ${ }^{148}$ P. Bortignon, ${ }^{148}$ D. Bourilkov, ${ }^{148}$ A. Brinkerhoff, ${ }^{148}$ L. Cadamuro, ${ }^{148}$ A. Carnes, ${ }^{148}$ M. Carver,${ }^{148}$ D. Curry, ${ }^{148}$ R. D. Field, ${ }^{148}$ S. V. Gleyzer, ${ }^{148}$ B. M. Joshi, ${ }^{148}$ J. Konigsberg, ${ }^{148}$ A. Korytov, ${ }^{148}$ P. Ma, ${ }^{148}$ K. Matchev, ${ }^{148}$ H. Mei, ${ }^{148}$ G. Mitselmakher, ${ }^{148}$ K. Shi, ${ }^{148}$ D. Sperka, ${ }^{148}$ J. Wang, ${ }^{148}$ S. Wang, ${ }^{148}$ Y. R. Joshi,${ }^{149}$ S. Linn, ${ }^{149}$ A. Ackert, ${ }^{150}$ T. Adams, ${ }^{150}$ A. Askew, ${ }^{150}$ S. Hagopian, ${ }^{150}$ V. Hagopian, ${ }^{150}$ K. F. Johnson, ${ }^{150}$ T. Kolberg, ${ }^{150}$ G. Martinez, ${ }^{150}$ T. Perry, ${ }^{150}$ H. Prosper, ${ }^{150}$ A. Saha, ${ }^{150}$ C. Schiber, ${ }^{150}$ V. Sharma, ${ }^{150}$ R. Yohay, ${ }^{150}$ M. M. Baarmand, ${ }^{151}$ V. Bhopatkar, ${ }^{151}$ S. Colafranceschi, ${ }^{151}$ M. Hohlmann, ${ }^{151}$ D. Noonan, ${ }^{151}$ M. Rahmani, ${ }^{151}$ T. Roy, ${ }^{151}$ F. Yumiceva,${ }^{151}$ M. R. Adams, ${ }^{152}$ L. Apanasevich,${ }^{152}$ D. Berry, ${ }^{152}$ R. R. Betts, ${ }^{152}$

R. Cavanaugh, ${ }^{152}$ X. Chen, ${ }^{152}$ S. Dittmer, ${ }^{152}$ O. Evdokimov,${ }^{152}$ C. E. Gerber, ${ }^{152}$ D. A. Hangal, ${ }^{152}$ D. J. Hofman, ${ }^{152}$ K. Jung, ${ }^{152}$ J. Kamin, ${ }^{152}$ C. Mills, ${ }^{152}$ I. D. Sandoval Gonzalez, ${ }^{152}$ M. B. Tonjes, ${ }^{152}$ N. Varelas, ${ }^{152}$ H. Wang, ${ }^{152}$ X. Wang, ${ }^{152}$ Z. Wu, ${ }^{152}$ J. Zhang, ${ }^{152}$ M. Alhusseini, ${ }^{153}$ B. Bilki, ${ }^{153, \text { qqq }}$ W. Clarida,${ }^{153}$ K. Dilsiz, ${ }^{153, \text { rrr }}$ S. Durgut, ${ }^{153}$ R. P. Gandrajula, ${ }^{153}$ M. Haytmyradov, ${ }^{153}$ V. Khristenko, ${ }^{153}$ J.-P. Merlo, ${ }^{153}$ A. Mestvirishvili, ${ }^{153}$ A. Moeller, ${ }^{153}$ J. Nachtman,${ }^{153}$ H. Ogul, ${ }^{153, s s s}$ Y. Onel, ${ }^{153}$ F. Ozok, ${ }^{153, \text { tt }}$ A. Penzo, ${ }^{153}$ C. Snyder, ${ }^{153}$ E. Tiras, ${ }^{153}$ J. Wetzel, ${ }^{153}$ B. Blumenfeld,,${ }^{154}$ A. Cocoros, ${ }^{154}$ N. Eminizer, ${ }^{154}$ D. Fehling, ${ }^{154}$ L. Feng, ${ }^{154}$ A. V. Gritsan, ${ }^{154}$ W. T. Hung, ${ }^{154}$ P. Maksimovic, ${ }^{154}$ J. Roskes,${ }^{154}$ U. Sarica, ${ }^{154}$ M. Swartz ${ }^{154}$ M. Xiao ${ }^{154}$ C. You, ${ }^{154}$ A. Al-bataineh,${ }^{155}$ P. Baringer, ${ }^{155}$ A. Bean, ${ }^{155}$ S. Boren, ${ }^{155}$ J. Bowen, ${ }^{155}$ A. Bylinkin, ${ }^{155}$ J. Castle, ${ }^{155}$ S. Khalil, ${ }^{155}$ A. Kropivnitskaya, ${ }^{155}$ D. Majumder, ${ }^{155}$ W. Mcbrayer, ${ }^{155}$ M. Murray, ${ }^{155}$ C. Rogan, ${ }^{155}$ S. Sanders, ${ }^{155}$ E. Schmitz, ${ }^{155}$ J. D. Tapia Takaki, ${ }^{155}$ Q. Wang, ${ }^{155}$ S. Duric, ${ }^{156}$ A. Ivanov, ${ }^{156}$ K. Kaadze, ${ }^{156}$ D. Kim, ${ }^{156}$ Y. Maravin, ${ }^{156}$

D. R. Mendis, ${ }^{156}$ T. Mitchell, ${ }^{156}$ A. Modak,${ }^{156}$ A. Mohammadi ${ }^{156}$ L. K. Saini, ${ }^{156}$ N. Skhirtladze, ${ }^{156}$ F. Rebassoo, ${ }^{157}$ D. Wright, ${ }^{157}$ A. Baden, ${ }^{158}$ O. Baron, ${ }^{158}$ A. Belloni, ${ }^{158}$ S. C. Eno, ${ }^{158}$ Y. Feng, ${ }^{158}$ C. Ferraioli, ${ }^{158}$ N. J. Hadley, ${ }^{158}$ S. Jabeen, ${ }^{158}$ G. Y. Jeng, ${ }^{158}$ R. G. Kellogg, ${ }^{158}$ J. Kunkle, ${ }^{158}$ A. C. Mignerey ${ }^{158}$ F. Ricci-Tam, ${ }^{158}$ Y. H. Shin, ${ }^{158}$ A. Skuja, ${ }^{158}$ S. C. Tonwar, ${ }^{158}$ K. Wong, ${ }^{158}$ D. Abercrombie, ${ }^{159}$ B. Allen, ${ }^{159}$ V. Azzolini, ${ }^{159}$ A. Baty,${ }^{159}$ G. Bauer, ${ }^{159}$ R. Bi, ${ }^{159}$ S. Brandt,${ }^{159}$ W. Busza, ${ }^{159}$ I. A. Cali, ${ }^{159}$ M. D’ Alfonso, ${ }^{159}$ Z. Demiragli, ${ }^{159}$ G. Gomez Ceballos, ${ }^{159}$ M. Goncharov, ${ }^{159}$ P. Harris, ${ }^{159}$ D. Hsu, ${ }^{159}$ M. Hu, ${ }^{159}$ Y. Iiyama, ${ }^{159}$ G. M. Innocenti ${ }^{159}$ M. Klute, ${ }^{159}$ D. Kovalskyi, ${ }^{159}$ Y.-J. Lee, ${ }^{159}$ P. D. Luckey, ${ }^{159}$ B. Maier, ${ }^{159}$ A. C. Marini, ${ }^{159}$ C. Mcginn, ${ }^{159}$ C. Mironov, ${ }^{159}$ S. Narayanan, ${ }^{159}$ X. Niu, ${ }^{159}$ C. Paus, ${ }^{159}$ C. Roland, ${ }^{159}$ G. Roland,${ }^{159}$ G. S. F. Stephans,${ }^{159}$ K. Sumorok, ${ }^{159}$ K. Tatar, ${ }^{159}$ D. Velicanu, ${ }^{159}$ J. Wang, ${ }^{159}$ T. W. Wang, ${ }^{159}$ B. Wyslouch, ${ }^{159}$ S. Zhaozhong, ${ }^{159}$ A. C. Benvenuti, ${ }^{160}$ R. M. Chatterjee, ${ }^{160}$ A. Evans, ${ }^{160}$ P. Hansen, ${ }^{160}$ S. Kalafut, ${ }^{160}$ Y. Kubota, ${ }^{160}$ Z. Lesko, ${ }^{160}$ J. Mans, ${ }^{160}$ N. Ruckstuhl, ${ }^{160}$ R. Rusack, ${ }^{160}$ J. Turkewitz, ${ }^{160}$ M. A. Wadud,${ }^{160}$ J. G. Acosta, ${ }^{161}$ S. Oliveros, ${ }^{161}$ E. Avdeeva, ${ }^{162}$ K. Bloom, ${ }^{162}$ D. R. Claes, ${ }^{162}$ C. Fangmeier, ${ }^{162}$ F. Golf, ${ }^{162}$ R. Gonzalez Suarez, ${ }^{162}$ R. Kamalieddin, ${ }^{162}$ I. Kravchenko, ${ }^{162}$ J. Monroy, ${ }^{162}$ J. E. Siado, ${ }^{162}$ G. R. Snow, ${ }^{162}$ B. Stieger, ${ }^{162}$ A. Godshalk, ${ }^{163}$ C. Harrington, ${ }^{163}$ I. Iashvili, ${ }^{163}$ A. Kharchilava, ${ }^{163}$ C. Mclean, ${ }^{163}$ D. Nguyen, ${ }^{163}$ A. Parker, ${ }^{163}$ S. Rappoccio, ${ }^{163}$ B. Roozbahani, ${ }^{163}$ G. Alverson, ${ }^{164}$ E. Barberis, ${ }^{164}$ C. Freer,${ }^{164}$ A. Hortiangtham, ${ }^{164}$ D. M. Morse, ${ }^{164}$ T. Orimoto, ${ }^{164}$ R. Teixeira De Lima, ${ }^{164}$ T. Wamorkar, ${ }^{164}$ B. Wang, ${ }^{164}$ A. Wisecarver, ${ }^{164}$ D. Wood, ${ }^{164}$ S. Bhattacharya, ${ }^{165}$ O. Charaf, ${ }^{165}$ K. A. Hahn, ${ }^{165}$ N. Mucia, ${ }^{165}$ N. Odell, ${ }^{165}$ M. H. Schmitt, ${ }^{165}$ S. Sevova, ${ }^{165}$ K. Sung, ${ }^{165}$ M. Trovato, ${ }^{165}$ M. Velasco, ${ }^{165}$ R. Bucci, ${ }^{166}$ N. Dev, ${ }^{166}$ M. Hildreth,${ }^{166}$ K. Hurtado Anampa ${ }^{166}$ C. Jessop, ${ }^{166}$ D. J. Karmgard, ${ }^{166}$ N. Kellams, ${ }^{166}$ K. Lannon, ${ }^{166}$ W. Li, ${ }^{166}$ N. Loukas, ${ }^{166}$ N. Marinelli, ${ }^{166}$ F. Meng, ${ }^{166}$ C. Mueller, ${ }^{166}$ Y. Musienko, ${ }^{166, \text { ii }}$ M. Planer, ${ }^{166}$ A. Reinsvold, ${ }^{166}$ R. Ruchti, ${ }^{166}$ P. Siddireddy, ${ }^{166}$ G. Smith, ${ }^{166}$ S. Taroni,${ }^{166}$ M. Wayne, ${ }^{166}$ A. Wightman, ${ }^{166}$ M. Wolf, ${ }^{166}$ A. Woodard, ${ }^{166}$ J. Alimena, ${ }^{167}$ L. Antonelli, ${ }^{167}$ B. Bylsma, ${ }^{167}$ L. S. Durkin, ${ }^{167}$ S. Flowers, ${ }^{167}$ B. Francis, ${ }^{167}$ A. Hart, ${ }^{167}$ C. Hill,,${ }^{167}$ W. Ji, ${ }^{167}$ T. Y. Ling, ${ }^{167}$ W. Luo, ${ }^{167}$ B. L. Winer, ${ }^{167}$ H. W. Wulsin, ${ }^{167}$ S. Cooperstein, ${ }^{168}$ P. Elmer, ${ }^{168}$ J. Hardenbrook, ${ }^{168}$ S. Higginbotham, ${ }^{168}$ A. Kalogeropoulos, ${ }^{168}$ D. Lange, ${ }^{168}$ M. T. Lucchini, ${ }^{168}$ J. Luo, ${ }^{168}$ D. Marlow, ${ }^{168}$ K. Mei, ${ }^{168}$ I. Ojalvo, ${ }^{168}$ J. Olsen, ${ }^{168}$ C. Palmer,${ }^{168}$ P. Piroué, ${ }^{168}$ J. Salfeld-Nebgen, ${ }^{168}$ D. Stickland, ${ }^{168}$ C. Tully, ${ }^{168}$ S. Malik, ${ }^{169}$ S. Norberg, ${ }^{169}$ A. Barker,${ }^{170}$ V. E. Barnes,${ }^{170}$ S. Das,${ }^{170}$ L. Gutay, ${ }^{170}$ M. Jones, ${ }^{170}$ A. W. Jung, ${ }^{170}$ A. Khatiwada, ${ }^{170}$ B. Mahakud, ${ }^{170}$ D. H. Miller, ${ }^{170}$ N. Neumeister, ${ }^{170}$ C. C. Peng, ${ }^{170}$ H. Qiu, ${ }^{170}$ J. F. Schulte ${ }^{170}$ J. Sun, ${ }^{170}$ F. Wang, ${ }^{170}$ R. Xiao, ${ }^{170}$ W. Xie, ${ }^{170}$ T. Cheng, ${ }^{171}$ J. Dolen, ${ }^{171}$ N. Parashar,${ }^{171}$ Z. Chen, ${ }^{172}$ K. M. Ecklund, ${ }^{172}$ S. Freed, ${ }^{172}$ F. J. M. Geurts, ${ }^{172}$ M. Kilpatrick, ${ }^{172}$ W. Li,${ }^{172}$ B. Michlin, ${ }^{172}$ B. P. Padley, ${ }^{172}$ J. Roberts, ${ }^{172}$ J. Rorie, ${ }^{172}$ W. Shi, ${ }^{172}$ Z. Tu, ${ }^{172}$ J. Zabel, ${ }^{172}$ A. Zhang, ${ }^{172}$ A. Bodek, ${ }^{173}$ P. de Barbaro, ${ }^{173}$ R. Demina, ${ }^{173}$ Y.t. Duh, ${ }^{173}$ J. L. Dulemba, ${ }^{173}$ C. Fallon, ${ }^{173}$ 
T. Ferbel, ${ }^{173}$ M. Galanti, ${ }^{173}$ A. Garcia-Bellido, ${ }^{173}$ J. Han, ${ }^{173}$ O. Hindrichs, ${ }^{173}$ A. Khukhunaishvili, ${ }^{173}$ K. H. Lo,${ }^{173}$ P. Tan, ${ }^{173}$ R. Taus, ${ }^{173}$ M. Verzetti, ${ }^{173}$ A. Agapitos, ${ }^{174}$ J. P. Chou, ${ }^{174}$ Y. Gershtein, ${ }^{174}$ T. A. Gómez Espinosa, ${ }^{174}$ E. Halkiadakis, ${ }^{174}$ M. Heindl, ${ }^{174}$ E. Hughes, ${ }^{174}$ S. Kaplan, ${ }^{174}$ R. Kunnawalkam Elayavalli, ${ }^{174}$ S. Kyriacou, ${ }^{174}$ A. Lath, ${ }^{174}$ R. Montalvo, ${ }^{174}$ K. Nash, ${ }^{174}$ M. Osherson, ${ }^{174}$ H. Saka, ${ }^{174}$ S. Salur, ${ }^{174}$ S. Schnetzer, ${ }^{174}$ D. Sheffield, ${ }^{174}$ S. Somalwar, ${ }^{174}$ R. Stone, ${ }^{174}$ S. Thomas, ${ }^{174}$ P. Thomassen, ${ }^{174}$ M. Walker ${ }^{174}$ A. G. Delannoy, ${ }^{175}$ J. Heideman,${ }^{175}$ G. Riley, ${ }^{175}$ S. Spanier, ${ }^{175}$ K. Thapa, ${ }^{175}$ O. Bouhali, ${ }^{176, \text { uuu }}$ A. Celik, ${ }^{176}$ M. Dalchenko, ${ }^{176}$ M. De Mattia, ${ }^{176}$ A. Delgado,${ }^{176}$ S. Dildick, ${ }^{176}$ R. Eusebi, ${ }^{176}$ J. Gilmore, ${ }^{176}$ T. Huang, ${ }^{176}$ T. Kamon, ${ }^{176, v v v}$ S. Luo, ${ }^{176}$ R. Mueller,${ }^{176}$ R. Patel, ${ }^{176}$ A. Perloff,,${ }^{176}$ L. Perniè, ${ }^{176}$ D. Rathjens, ${ }^{176}$ A. Safonov, ${ }^{176}$ N. Akchurin, ${ }^{177}$ J. Damgov, ${ }^{177}$ F. De Guio, ${ }^{177}$ P. R. Dudero, ${ }^{177}$ S. Kunori, ${ }^{177}$ K. Lamichhane, ${ }^{177}$ S. W. Lee, ${ }^{177}$ T. Mengke, ${ }^{177}$ S. Muthumuni, ${ }^{177}$ T. Peltola, ${ }^{177}$ S. Undleeb, ${ }^{177}$ I. Volobouev, ${ }^{177}$ Z. Wang, ${ }^{177}$ S. Greene, ${ }^{178}$ A. Gurrola, ${ }^{178}$ R. Janjam, ${ }^{178}$ W. Johns, ${ }^{178}$ C. Maguire, ${ }^{178}$ A. Melo ${ }^{178}$ H. Ni, ${ }^{178}$ K. Padeken, ${ }^{178}$ J. D. Ruiz Alvarez ${ }^{178}$ P. Sheldon, ${ }^{178}$ S. Tuo, ${ }^{178}$ J. Velkovska, ${ }^{178}$ M. Verweij, ${ }^{178}$ Q. Xu,${ }^{178}$ M. W. Arenton, ${ }^{179}$ P. Barria, ${ }^{179}$ B. Cox,${ }^{179}$ R. Hirosky, ${ }^{179}$ M. Joyce, ${ }^{179}$ A. Ledovskoy, ${ }^{179} \mathrm{H}$. Li, ${ }^{179}$ C. Neu, ${ }^{179}$ T. Sinthuprasith, ${ }^{179}$ Y. Wang, ${ }^{179}$ E. Wolfe,${ }^{179}$ F. Xia, ${ }^{179}$ R. Harr, ${ }^{180}$ P. E. Karchin, ${ }^{180}$ N. Poudyal, ${ }^{180}$ J. Sturdy, ${ }^{180}$ P. Thapa ${ }^{180}$ S. Zaleski, ${ }^{180}$ M. Brodski, ${ }^{181}$ J. Buchanan, ${ }^{181}$ C. Caillol, ${ }^{181}$ D. Carlsmith,${ }^{181}$ S. Dasu, ${ }^{181}$ L. Dodd ${ }^{181}$ B. Gomber ${ }^{181}$ M. Grothe ${ }^{181}$ M. Herndon, ${ }^{181}$ A. Hervé,${ }^{181}$ U. Hussain, ${ }^{181}$ P. Klabbers, ${ }^{181}$ A. Lanaro, ${ }^{181}$ K. Long, ${ }^{181}$ R. Loveless, ${ }^{181}$ T. Ruggles, ${ }^{181}$ A. Savin, ${ }^{181}$ N. Smith, ${ }^{181}$ W. H. Smith, ${ }^{181}$ and N. Woods ${ }^{181}$

(CMS Collaboration)

\author{
${ }^{1}$ Yerevan Physics Institute, Yerevan, Armenia \\ ${ }^{2}$ Institut für Hochenergiephysik, Wien, Austria \\ ${ }^{3}$ Institute for Nuclear Problems, Minsk, Belarus \\ ${ }^{4}$ Universiteit Antwerpen, Antwerpen, Belgium \\ ${ }^{5}$ Vrije Universiteit Brussel, Brussel, Belgium \\ ${ }^{6}$ Université Libre de Bruxelles, Bruxelles, Belgium \\ ${ }^{7}$ Ghent University, Ghent, Belgium \\ ${ }^{8}$ Université Catholique de Louvain, Louvain-la-Neuve, Belgium \\ ${ }^{9}$ Centro Brasileiro de Pesquisas Fisicas, Rio de Janeiro, Brazil \\ ${ }^{10}$ Universidade do Estado do Rio de Janeiro, Rio de Janeiro, Brazil \\ ${ }^{11 a}$ Universidade Estadual Paulista, São Paulo, Brazil \\ ${ }^{11 \mathrm{~b}}$ Universidade Federal do ABC, São Paulo, Brazil \\ ${ }^{12}$ Institute for Nuclear Research and Nuclear Energy, Bulgarian Academy of Sciences, Sofia, Bulgaria \\ ${ }^{13}$ University of Sofia, Sofia, Bulgaria \\ ${ }^{14}$ Beihang University, Beijing, China \\ ${ }^{15}$ Institute of High Energy Physics, Beijing, China \\ ${ }^{16}$ State Key Laboratory of Nuclear Physics and Technology, Peking University, Beijing, China \\ ${ }^{17}$ Tsinghua University, Beijing, China \\ ${ }^{18}$ Universidad de Los Andes, Bogota, Colombia \\ ${ }^{19}$ University of Split, Faculty of Electrical Engineering, Mechanical Engineering and Naval Architecture, Split, Croatia \\ ${ }^{20}$ University of Split, Faculty of Science, Split, Croatia \\ ${ }^{21}$ Institute Rudjer Boskovic, Zagreb, Croatia \\ ${ }^{22}$ University of Cyprus, Nicosia, Cyprus \\ ${ }^{23}$ Charles University, Prague, Czech Republic \\ ${ }^{24}$ Escuela Politecnica Nacional, Quito, Ecuador \\ ${ }^{25}$ Universidad San Francisco de Quito, Quito, Ecuador \\ ${ }^{26}$ Academy of Scientific Research and Technology of the Arab Republic of Egypt, Egyptian Network of High Energy Physics, \\ Cairo, Egypt \\ ${ }^{27}$ National Institute of Chemical Physics and Biophysics, Tallinn, Estonia \\ ${ }^{28}$ Department of Physics, University of Helsinki, Helsinki, Finland \\ ${ }^{29}$ Helsinki Institute of Physics, Helsinki, Finland \\ ${ }^{30}$ Lappeenranta University of Technology, Lappeenranta, Finland \\ ${ }^{31}$ IRFU, CEA, Université Paris-Saclay, Gif-sur-Yvette, France \\ ${ }^{32}$ Laboratoire Leprince-Ringuet, Ecole polytechnique, CNRS/IN2P3, Université Paris-Saclay, Palaiseau, France \\ ${ }^{33}$ Université de Strasbourg, CNRS, IPHC UMR 7178, Strasbourg, France
}


${ }^{34}$ Centre de Calcul de l'Institut National de Physique Nucleaire et de Physique des Particules, CNRS/IN2P3, Villeurbanne, France

${ }^{35}$ Université de Lyon, Université Claude Bernard Lyon 1, CNRS-IN2P3, Institut de Physique Nucléaire de Lyon, Villeurbanne, France

${ }^{36}$ Georgian Technical University, Tbilisi, Georgia

${ }^{37}$ Tbilisi State University, Tbilisi, Georgia

${ }^{38}$ RWTH Aachen University, I. Physikalisches Institut, Aachen, Germany

${ }^{39}$ RWTH Aachen University, III. Physikalisches Institut A, Aachen, Germany

${ }^{40}$ RWTH Aachen University, III. Physikalisches Institut B, Aachen, Germany

${ }^{41}$ Deutsches Elektronen-Synchrotron, Hamburg, Germany

${ }^{42}$ University of Hamburg, Hamburg, Germany

${ }^{43}$ Karlsruher Institut fuer Technology

${ }^{44}$ Institute of Nuclear and Particle Physics (INPP), NCSR Demokritos, Aghia Paraskevi, Greece

${ }^{45}$ National and Kapodistrian University of Athens, Athens, Greece

${ }^{46}$ National Technical University of Athens, Athens, Greece

${ }^{47}$ University of Ioánnina, Ioánnina, Greece

${ }^{48}$ MTA-ELTE Lendület CMS Particle and Nuclear Physics Group, Eötvös Loránd University, Budapest, Hungary

${ }^{49}$ Wigner Research Centre for Physics, Budapest, Hungary

${ }^{50}$ Institute of Nuclear Research ATOMKI, Debrecen, Hungary

${ }^{51}$ Institute of Physics, University of Debrecen, Debrecen, Hungary

${ }^{52}$ Indian Institute of Science (IISc), Bangalore, India

${ }^{53}$ National Institute of Science Education and Research, HBNI, Bhubaneswar, India

${ }^{54}$ Panjab University, Chandigarh, India

${ }^{55}$ University of Delhi, Delhi, India

${ }^{56}$ Saha Institute of Nuclear Physics, HBNI, Kolkata,India

${ }^{57}$ Indian Institute of Technology Madras, Madras, India

${ }^{58}$ Bhabha Atomic Research Centre, Mumbai, India

${ }^{59}$ Tata Institute of Fundamental Research-A, Mumbai, India

${ }^{60}$ Tata Institute of Fundamental Research-B, Mumbai, India

${ }^{61}$ Indian Institute of Science Education and Research (IISER), Pune, India

${ }^{62}$ Institute for Research in Fundamental Sciences (IPM), Tehran, Iran

${ }^{63}$ University College Dublin, Dublin, Ireland

${ }^{64 a}$ INFN Sezione di Bari, Bari, Italy

${ }^{64 \mathrm{~b}}$ Università di Bari, Bari, Italy

${ }^{64 \mathrm{c}}$ Politecnico di Bari, Bari, Italy

${ }^{65 \mathrm{a}}$ INFN Sezione di Bologna, Bologna, Italy

${ }^{65 \mathrm{~b}}$ Università di Bologna, Bologna, Italy

${ }^{66 a}$ INFN Sezione di Catania, Catania, Italy

${ }^{66 \mathrm{~b}}$ Università di Catania, Catania, Italy

${ }^{67 \mathrm{a}}$ INFN Sezione di Firenze, Firenze, Italy

${ }^{67 \mathrm{~b}}$ Università di Firenze, Firenze, Italy

${ }^{68}$ INFN Laboratori Nazionali di Frascati, Frascati, Italy

${ }^{69 a}$ INFN Sezione di Genova, Genova, Italy

${ }^{69 b}$ Università di Genova, Genova, Italy

${ }^{70 a}$ INFN Sezione di Milano-Bicocca, Milano, Italy

${ }^{70 \mathrm{~b}}$ Università di Milano-Bicocca, Milano, Italy

${ }^{71 a}$ INFN Sezione di Napoli, Napoli, Italy

${ }^{71 \mathrm{~b}}$ Università di Napoli 'Federico II', Napoli, Italy

${ }^{71 \mathrm{c}}$ Università della Basilicata, Potenza, Italy

${ }^{71 d}$ Università G. Marconi, Roma, Italy

${ }^{72 a}$ INFN Sezione di Padova, Padova, Italy

${ }^{72 \mathrm{~b}}$ Università di Padova, Padova, Italy

${ }^{72 \mathrm{c}}$ Università di Trento, Trento, Italy

${ }^{73 a}$ INFN Sezione di Pavia

${ }^{73 b}$ Università di Pavia

${ }^{74 \mathrm{a}}$ INFN Sezione di Perugia, Perugia, Italy

${ }^{74 \mathrm{~b}}$ Università di Perugia, Perugia, Italy

${ }^{75 a}$ INFN Sezione di Pisa, Pisa, Italy

${ }^{75 b}$ Università di Pisa, Pisa, Italy 
${ }^{75 c}$ Scuola Normale Superiore di Pisa, Pisa, Italy

${ }^{76 a}$ INFN Sezione di Roma, Rome, Italy

${ }^{76 \mathrm{~b}}$ Sapienza Università di Roma, Rome, Italy

${ }^{77 a}$ INFN Sezione di Torino, Torino, Italy

${ }^{77 b}$ Università di Torino, Torino, Italy

${ }^{77 \mathrm{c}}$ Università del Piemonte Orientale, Novara, Italy

${ }^{78 a}$ INFN Sezione di Trieste, Trieste, Italy

${ }^{78 b}$ Università di Trieste, Trieste, Italy

${ }^{79}$ Kyungpook National University

${ }^{80}$ Chonnam National University, Institute for Universe and Elementary Particles, Kwangju, Korea

${ }^{81}$ Hanyang University, Seoul, Korea

${ }^{82}$ Korea University, Seoul, Korea

${ }^{83}$ Sejong University, Seoul, Korea

${ }^{84}$ Seoul National University, Seoul, Korea

${ }^{85}$ University of Seoul, Seoul, Korea

${ }^{86}$ Sungkyunkwan University, Suwon, Korea

${ }^{87}$ Vilnius University, Vilnius, Lithuania

${ }^{88}$ National Centre for Particle Physics, Universiti Malaya, Kuala Lumpur, Malaysia

${ }^{89}$ Universidad de Sonora (UNISON), Hermosillo, Mexico

${ }^{90}$ Centro de Investigacion y de Estudios Avanzados del IPN, Mexico City, Mexico

${ }^{91}$ Universidad Iberoamericana, Mexico City, Mexico

${ }^{92}$ Benemerita Universidad Autonoma de Puebla, Puebla, Mexico

${ }^{93}$ Universidad Autónoma de San Luis Potosí, San Luis Potosí, Mexico

${ }^{94}$ University of Auckland, Auckland, New Zealand

${ }^{95}$ University of Canterbury, Christchurch, New Zealand

${ }^{96}$ National Centre for Physics, Quaid-I-Azam University, Islamabad, Pakistan

${ }^{97}$ National Centre for Nuclear Research, Swierk, Poland

${ }^{98}$ Institute of Experimental Physics, Faculty of Physics, University of Warsaw, Warsaw, Poland

${ }^{99}$ Laboratório de Instrumentação e Física Experimental de Partículas, Lisboa, Portugal

${ }^{100}$ Joint Institute for Nuclear Research, Dubna, Russia

${ }^{101}$ Petersburg Nuclear Physics Institute, Gatchina (St. Petersburg), Russia

${ }^{102}$ Institute for Nuclear Research, Moscow, Russia

${ }^{103}$ Institute for Theoretical and Experimental Physics, Moscow, Russia

${ }^{104}$ Moscow Institute of Physics and Technology, Moscow, Russia

${ }^{105}$ National Research Nuclear University 'Moscow Engineering Physics Institute' (MEPhI), Moscow, Russia

${ }^{106}$ P.N. Lebedev Physical Institute, Moscow, Russia

${ }^{107}$ Skobeltsyn Institute of Nuclear Physics, Lomonosov Moscow State University, Moscow, Russia

${ }^{108}$ Novosibirsk State University (NSU), Novosibirsk, Russia

${ }^{109}$ State Research Center of Russian Federation, Institute for High Energy Physics of NRC “Kurchatov Institute”, Protvino, Russia

${ }^{110}$ National Research Tomsk Polytechnic University, Tomsk, Russia

${ }^{111}$ University of Belgrade, Faculty of Physics and Vinca Institute of Nuclear Sciences, Belgrade, Serbia

${ }^{112}$ Centro de Investigaciones Energéticas Medioambientales y Tecnológicas (CIEMAT), Madrid, Spain

${ }^{113}$ Universidad Autónoma de Madrid, Madrid, Spain

${ }^{114}$ Universidad de Oviedo, Oviedo, Spain

${ }^{115}$ Instituto de Física de Cantabria (IFCA), CSIC-Universidad de Cantabria, Santander, Spain

${ }^{116}$ CERN, European Organization for Nuclear Research, Geneva, Switzerland

${ }^{117}$ Paul Scherrer Institut, Villigen, Switzerland

${ }^{118}$ ETH Zurich - Institute for Particle Physics and Astrophysics (IPA), Zurich, Switzerland

${ }^{119}$ Universität Zürich, Zurich, Switzerland

${ }^{120}$ National Central University, Chung-Li, Taiwan

${ }^{121}$ National Taiwan University (NTU), Taipei, Taiwan

${ }^{122}$ Chulalongkorn University, Faculty of Science, Department of Physics, Bangkok, Thailand

${ }^{123}$ Cukurova University, Physics Department, Science and Art Faculty, Adana, Turkey

${ }^{124}$ Middle East Technical University, Physics Department, Ankara, Turkey

${ }^{125}$ Bogazici University, Istanbul, Turkey

${ }^{126}$ Istanbul Technical University, Istanbul, Turkey

${ }^{127}$ Institute for Scintillation Materials of National Academy of Science of Ukraine, Kharkov, Ukraine

${ }^{128}$ National Scientific Center, Kharkov Institute of Physics and Technology, Kharkov, Ukraine

${ }^{129}$ University of Bristol, Bristol, United Kingdom 
${ }^{130}$ Rutherford Appleton Laboratory, Didcot, United Kingdom

${ }^{131}$ Imperial College, London, United Kingdom

${ }^{132}$ Brunel University, Uxbridge, United Kingdom

${ }^{133}$ Baylor University, Waco, Texas, USA

${ }^{134}$ Catholic University of America, Washington, DC, USA

${ }^{135}$ The University of Alabama, Tuscaloosa, Alabama, USA

${ }^{136}$ Boston University, Boston, Massachusetts, USA

${ }^{137}$ Brown University, Providence, Rhode Island, USA

${ }^{138}$ University of California at Davis, Davis, California, USA

${ }^{139}$ University of California at Los Angeles, Los Angeles, California, USA

${ }^{140}$ University of California at Riverside, Riverside, California, USA

${ }^{141}$ University of California at San Diego, La Jolla, California, USA

${ }^{142}$ University of California at Santa Barbara, Department of Physics, Santa Barbara, California, USA

${ }^{143}$ California Institute of Technology, Pasadena, California, USA

${ }^{144}$ Carnegie Mellon University, Pittsburgh, Pennsylvania, USA

${ }^{145}$ University of Colorado Boulder, Boulder, Colorado, USA

${ }^{146}$ Cornell University, Ithaca, New York, USA

${ }^{147}$ Fermi National Accelerator Laboratory, Batavia, Illinois, USA

${ }^{148}$ University of Florida, Gainesville, Florida, USA

${ }^{149}$ Florida International University, Miami, Florida, USA

${ }^{150}$ Florida State University, Tallahassee, Florida, USA

${ }^{151}$ Florida Institute of Technology, Melbourne, Florida, USA

${ }^{152}$ University of Illinois at Chicago (UIC), Chicago, Illinois, USA

${ }^{153}$ The University of Iowa, Iowa City, Iowa, USA

${ }^{154}$ Johns Hopkins University, Baltimore, Maryland, USA

${ }^{155}$ The University of Kansas, Lawrence, Kansas, USA

${ }^{156}$ Kansas State University, Manhattan, Kansas, USA

${ }^{157}$ Lawrence Livermore National Laboratory, Livermore, California, USA

${ }^{158}$ University of Maryland, College Park, Maryland, USA

${ }^{159}$ Massachusetts Institute of Technology, Cambridge, Massachusetts, USA

${ }^{160}$ University of Minnesota, Minneapolis, Minnesota, USA

${ }^{161}$ University of Mississippi, Oxford, Mississippi, USA

${ }^{162}$ University of Nebraska-Lincoln, Lincoln, Nebraska, USA

${ }^{163}$ State University of New York at Buffalo, Buffalo, New York, USA

${ }^{164}$ Northeastern University, Boston, Massachusetts, USA

${ }^{165}$ Northwestern University, Evanston, Illinois, USA

${ }^{166}$ University of Notre Dame, Notre Dame, Indiana, USA

${ }^{167}$ The Ohio State University, Columbus, Ohio, USA

${ }^{168}$ Princeton University, Princeton, New Jersey, USA

${ }^{169}$ University of Puerto Rico, Mayaguez, Puerto Rico

${ }^{170}$ Purdue University, West Lafayette, Indiana, USA

${ }^{171}$ Purdue University Northwest, Hammond, Indiana, USA

${ }^{172}$ Rice University, Houston, Texas, USA

${ }^{173}$ University of Rochester, Rochester, New York, USA

${ }^{174}$ Rutgers, The State University of New Jersey, Piscataway, New Jersey, USA

${ }^{175}$ University of Tennessee, Knoxville, Tennessee, USA

${ }^{176}$ Texas A\&M University, College Station, Texas, USA

${ }^{177}$ Texas Tech University, Lubbock, Texas, USA

${ }^{178}$ Vanderbilt University, Nashville, Tennessee, USA

${ }^{179}$ University of Virginia, Charlottesville, Virginia, USA

${ }^{180}$ Wayne State University, Detroit, Michigan, USA

${ }^{181}$ University of Wisconsin - Madison, Madison, Wisconsin, USA

\footnotetext{
${ }^{\mathrm{a}}$ Deceased.

${ }^{\mathrm{b}}$ Also at Vienna University of Technology, Vienna, Austria.

${ }^{c}$ Also at IRFU, CEA, Université Paris-Saclay, Gif-sur-Yvette, France.

${ }^{\mathrm{d}}$ Also at Universidade Estadual de Campinas, Campinas, Brazil.

${ }^{\mathrm{e}}$ Also at Federal University of Rio Grande do Sul, Porto Alegre, Brazil.

${ }^{\mathrm{f}}$ Also at Université Libre de Bruxelles, Bruxelles, Belgium.

${ }^{\mathrm{g}}$ Also at University of Chinese Academy of Sciences, Beijing, China.
} 
${ }^{\mathrm{h}}$ Also at Institute for Theoretical and Experimental Physics, Moscow, Russia.

${ }^{\mathrm{i}}$ Also at Joint Institute for Nuclear Research, Dubna, Russia.

${ }^{\mathrm{j}}$ Also at British University in Egypt, Cairo, Egypt.

${ }^{k}$ Also at Suez University, Suez, Egypt.

${ }^{1}$ Also at Fayoum University, El-Fayoum, Egypt.

${ }^{\mathrm{m}}$ Also at Department of Physics, King Abdulaziz University, Jeddah, Saudi Arabia.

${ }^{\mathrm{n}}$ Also at Université de Haute Alsace, Mulhouse, France.

${ }^{\circ}$ Also at Skobeltsyn Institute of Nuclear Physics, Lomonosov Moscow State University, Moscow, Russia.

${ }^{\mathrm{p}}$ Also at CERN, European Organization for Nuclear Research, Geneva, Switzerland.

${ }^{\mathrm{q}}$ Also at RWTH Aachen University, III. Physikalisches Institut A, Aachen, Germany.

${ }^{\mathrm{r}}$ Also at University of Hamburg, Hamburg, Germany.

${ }^{\mathrm{s}}$ Also at Brandenburg University of Technology, Cottbus, Germany.

tAlso at MTA-ELTE Lendület CMS Particle and Nuclear Physics Group, Eötvös Loránd University, Budapest, Hungary.

"Also at Institute of Nuclear Research ATOMKI, Debrecen, Hungary.

${ }^{v}$ Also at Institute of Physics, University of Debrecen, Debrecen, Hungary.

${ }^{\mathrm{w}}$ Also at IIT Bhubaneswar, Bhubaneswar, India.

${ }^{\mathrm{x}}$ Also at Institute of Physics, Bhubaneswar, India.

${ }^{\mathrm{y}}$ Also at Shoolini University, Solan, India.

${ }^{\mathrm{z}}$ Also at University of Visva-Bharati, Santiniketan, India.

${ }^{\text {aa } A l s o ~ a t ~ I s f a h a n ~ U n i v e r s i t y ~ o f ~ T e c h n o l o g y, ~ I s f a h a n, ~ I r a n . ~}$

${ }^{\mathrm{bb}}$ Also at Plasma Physics Research Center, Science and Research Branch, Islamic Azad University, Tehran, Iran.

${ }^{\mathrm{cc}}$ Also at Università degli Studi di Siena, Siena, Italy.

${ }^{\mathrm{dd}}$ Also at Kyunghee University, Seoul, Korea.

${ }^{e e}$ Also at International Islamic University of Malaysia, Kuala Lumpur, Malaysia.

${ }^{\mathrm{ff}}$ Also at Malaysian Nuclear Agency, MOSTI, Kajang, Malaysia.

${ }^{g g}$ Also at Consejo Nacional de Ciencia y Tecnología, Mexico city, Mexico.

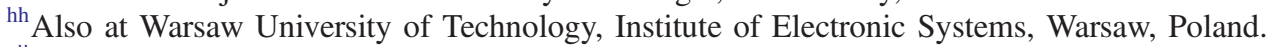

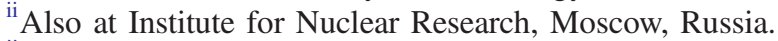

${ }^{\mathrm{ij}}$ Also at National Research Nuclear University 'Moscow Engineering Physics Institute' (MEPhI), Moscow, Russia.

${ }^{k k}$ Also at St. Petersburg State Polytechnical University, St. Petersburg, Russia.

"Also at University of Florida, Gainesville, Florida, USA.

${ }^{\mathrm{mm}}$ Also at P.N. Lebedev Physical Institute, Moscow, Russia.

${ }^{\mathrm{nn}}$ Also at California Institute of Technology, Pasadena, California, USA.

${ }^{00}$ Also at Budker Institute of Nuclear Physics, Novosibirsk, Russia.

${ }^{\mathrm{pp}}$ Also at Faculty of Physics, University of Belgrade, Belgrade, Serbia.

${ }^{\mathrm{qq}}$ Also at INFN Sezione di Pavia, Università di Pavia, Pavia, Italy.

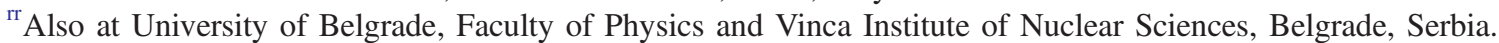

${ }^{\text {ss }}$ Also at Scuola Normale e Sezione dell'INFN, Pisa, Italy.

${ }^{\text {tt} A l s o ~ a t ~ N a t i o n a l ~ a n d ~ K a p o d i s t r i a n ~ U n i v e r s i t y ~ o f ~ A t h e n s, ~ A t h e n s, ~ G r e e c e . ~}$

${ }^{\text {uu }}$ Also at Riga Technical University, Riga, Latvia.

${ }^{\mathrm{vv}}$ Also at Universität Zürich, Zurich, Switzerland.

${ }^{\text {ww }}$ Also at Stefan Meyer Institute for Subatomic Physics (SMI), Vienna, Austria.

${ }^{\mathrm{xx}}$ Also at Adiyaman University, Adiyaman, Turkey.

${ }^{\mathrm{yy}}$ Also at Istanbul Aydin University, Istanbul, Turkey.

${ }^{\mathrm{zz}}$ Also at Mersin University, Mersin, Turkey.

${ }^{a a a}$ Also at Piri Reis University, Istanbul, Turkey.

${ }^{b b b}$ Also at Gaziosmanpasa University, Tokat, Turkey.

${ }^{c c c}$ Also at Ozyegin University, Istanbul, Turkey.

ddd Also at Izmir Institute of Technology, Izmir, Turkey.

${ }^{\text {eee }}$ Also at Marmara University, Istanbul, Turkey.

${ }^{\text {fff }}$ Also at Kafkas University, Kars, Turkey.

${ }^{\text {ggg }}$ Also at Istanbul University, Faculty of Science, Istanbul, Turkey.

hhh Also at Istanbul Bilgi University, Istanbul, Turkey.

iii Also at Hacettepe University, Ankara, Turkey.

${ }^{\mathrm{ijj}}$ Also at Rutherford Appleton Laboratory, Didcot, United Kingdom.

${ }^{k k k}$ Also at School of Physics and Astronomy, University of Southampton, Southampton, United Kingdom.

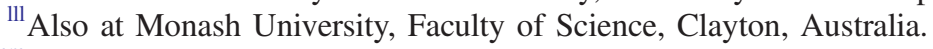

${ }^{\mathrm{mmm}}$ Also at Bethel University, St. Paul, Minnesota, USA.

${ }^{n n n}$ Also at Karamanoğlu Mehmetbey University, Karaman, Turkey.

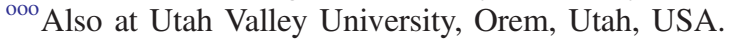


${ }^{\mathrm{ppp}}$ Also at Purdue University, West Lafayette, Indiana, USA.

${ }^{\mathrm{qqq}}$ Also at Beykent University, Istanbul, Turkey.

${ }^{\mathrm{rrr}}$ Also at Bingol University, Bingol, Turkey.

${ }^{\text {sss }}$ Also at Sinop University, Sinop, Turkey.

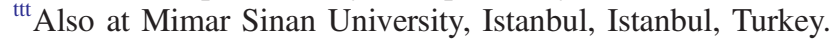

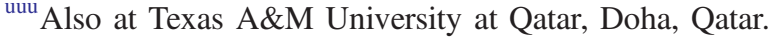

${ }^{\mathrm{vvv}}$ Also at Kyungpook National University, Daegu, Korea. 